\title{
Cardiovascular Events and Mortality in Patients Undergoing Adjuvant Radiotherapy for Breast Cancer: a Systematic Review
}

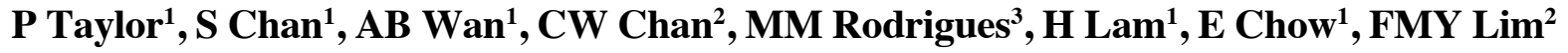 \\ ${ }^{1}$ Odette Cancer Centre, Sunnybrook Health Sciences Centre, University of Toronto, Toronto, Ontario, Canada \\ ${ }^{2}$ Department of Oncology, Princess Margaret Hospital, Hong Kong \\ ${ }^{3}$ Centro Oncológico AZ do Noroeste, Patos de Minas, Minas Gerais, Brazil
}

\begin{abstract}
Objective: We performed a systematic review to quantify the cardiovascular risk of adjuvant radiotherapy $(R T)$ for breast cancer.

Methods: A literature search was conducted using MEDLINE, Embase, and the Cochrane Central Register of Controlled Trials from inception to July 2020.

Results: The literature search produced 7363 reports, of which 76 met our inclusion criteria. In studies comparing left-sided RT with right-sided RT, 7 of 35 (20\%) studies found increased cardiovascular mortality, and 8 of $28(29 \%)$ studies found increased cardiovascular events. In studies comparing patients who received RT with those who did not, 7 of 26 (27\%) studies found increased cardiovascular mortality, and 5 of 22 (23\%) studies found increased cardiovascular events.

Conclusion: Most of the studies that found significant associations between laterality and cardiovascular risks included treatment periods that started prior to 1985, suggesting that modern RT techniques have minimised the cardiac exposure in breast cancer patients receiving RT. However, more focused studies must be conducted to investigate the long-term cardiovascular risk associated with modern RT techniques.
\end{abstract}

Key Words: Breast neoplasms; Heart disease risk factors; Morbidity; Mortality; Radiotherapy, adjuvant

\section{中文摘要}

\section{乳腺癌輔助放療患者的心血管疾病和死亡率 : 系統性文獻回顧 \\ P Taylor、S Chan、AB Wan、陳俊尹、MM Rodrigues、H Lam、E Chow、林美瑩 \\ 目的 : 我們進行系統性文獻回顧，量化乳腺癌術後輔助放療的心血管疾病風險。}

\footnotetext{
Correspondence: Dr FMY Lim, Department of Oncology, Princess Margaret Hospital, Hong Kong

Email:lmy084@ha.org.hk
}

Submitted: 21 May 2020; Accepted: 15 Jan 2021

Contributors: PT, SC, ABW, and EC designed the study. PT, ABW, and HL acquired the data. PT, SC, ABW, CWC, MMR, and FMYL analysed the data. PT and SC drafted the manuscript. All authors critically revised the manuscript for important intellectual content. All authors had full access to the data, contributed to the study, approved the final version for publication, and take responsibility for its accuracy and integrity.

Conflicts of Interest: All authors have disclosed no conflicts of interest.

Funding/Support: This research received no specific grant from any funding agency in the public, commercial, or not-for-profit sectors.

Data Availability: All data generated or analysed during the present study are available from the corresponding author on reasonable request.

Ethics Approval: Ethics approval was not required for this review article which does not involve patients / animal or any interventions. 
方法：使用 MEDLINE、Embase和Cochrane Central Register of Controlled Trials檢索始至2020年7月刊登 的文獻。

結果 : 文獻檢索出7363個結果, 其中76個符合我們的納入標準。在比較左側乳腺癌放療與右側乳 腺癌放療的研究中, 35項研究中有 7 項 $(20 \%)$ 發現心血管疾病死亡率增加, 28 項研究中有 8 項 (29\%) 發現心血管疾病增加。在比較接受放療的患者與未接受放療的患者的研究中, 26項研究中 有 7項 $(27 \%)$ 發現心血管疾病死亡率增加, 22項研究中有 5項 $(23 \%)$ 發現心血管疾病增加。

結論 : 大部份發現單側性乳癌與心血管疾病風險間存在顯著關聯的研究都是1985年或之前, 這表明 現代放療技術已將接受放療的乳腺癌患者的心臟暴露風險降至最低。然而, 必須進行更有針對性的 研究以檢視與現代放療技術相關的長期心血管疾病風險。

\section{INTRODUCTION}

Breast cancer is the most frequently diagnosed cancer and the leading cause of cancer death in females worldwide. ${ }^{1}$ It has been shown through randomised trials that adjuvant radiotherapy (RT) following breast-conserving surgery substantially reduces breast cancer recurrence and reduces the absolute breast cancer mortality rate., ${ }^{2,3}$ RT administered to breast cancer patients usually exposes the heart, an organ at risk, to some radiation. Cheng et $\mathrm{al}^{4}$ conducted a literature review and metaanalysis on this topic, including studies published prior to January 2015, and found that breast cancer RT was associated with an absolute increase of 76.4 cases of coronary heart disease $(95 \%$ confidence interval $[\mathrm{CI}]=36.8-130.5)$ and 125.5 cases of cardiac death (95\% CI=98.8-157.9) per 100000 person-years, respectively. In order to create optimised and tailored treatment plans, the current relationship between adjuvant breast RT and cardiovascular risks must be studied so that physicians and patients may appropriately consider the benefits of reduced breast cancer mortality with the potential long-term cardiovascular risks. We performed a systematic review to assess the risk of cardiovascular events (CVEs) and cardiovascular mortality (CVM) and its correlation with breast/chest wall RT for women with breast cancer (including breast cancer and ductal carcinoma in situ) following breast-conserving surgery/ mastectomy (for node-positive or involved resection margin disease), as well as disease laterality. This will allow radiation oncologists to better inform their patients about the risks and benefits of adjuvant RT so that patients may make a more informed decision.

\section{METHODS}

\section{Search Strategy}

A literature search was completed using MEDLINE, Embase, and Cochrane Central Register of Controlled
Trials from inception through to July 2020. Search terms for breast cancer included 'breast cancer', 'breast neoplasm', 'breast carcinoma', and 'breast tumour or tumour' (online supplementary Appendix). RT terms included 'radiotherapy', 'radiation', 'irradiation', and 'radiation injury'. CVE terms included 'heart disease', 'heart infarction', 'myocardial infarction (MI) or heart attack', 'angina pectoris', 'congestive heart failure (CHF)', 'coronary artery disease (CAD)', 'coronary artery obstruction', 'heart or cardio or cardiovascular disease (CVD)', 'ischemic heart disease (IHD)', 'dosage risk', 'cardiovascular risk', and 'cardiovascular death'.

\section{Study Selection}

Screening was first done based on the title and abstract independently by two authors (P Taylor, S Chan), with discrepancies being resolved through discussion between the two authors. Then, full-text screening was conducted independently by the two authors. Inclusion criteria were reports of the clinical cardiovascular outcomes, including CVEs and/or CVM as defined above. Specifically, studies were included if they reported comparisons in clinical cardiovascular outcomes between patients who received RT and those who did not receive RT and/or between patients who received left-sided RT and those who received right-sided RT. Exclusion criteria included any studies that investigated cancers other than breast cancer and the effects of irradiation on organ systems other than the cardiovascular system. Studies employing brachytherapy, partial breast irradiation, or boost to the tumour bed alone were excluded. Full-length papers, including cohort studies, case-control studies, and randomised controlled trials published as original papers written in English, were considered. Any case reports and non-original articles such as systematic reviews were excluded. 


\section{Data Collection and Analysis}

Data extraction was conducted independently by two authors (P Taylor, S Chan). Both authors engaged in a discussion regarding any discrepancies between the extracted data and came to a consensus. The following data were extracted from the papers: publication year, geographical location, sample size, mean/median follow-up, number of CVEs, number of cardiovascular deaths, laterality, hazard ratios (HRs), incidence ratios, risk ratios (RRs), mortality ratios (MRs), and associated measures of variance for all categories of outcomes.

\section{RESULTS}

The search identified 7363 publications, of which 1063 were duplicates and excluded (Figure). A further 6132 articles were excluded because they did not meet the inclusion criteria. The remaining 168 articles underwent full-text screening. Of them, 92 were excluded for failure to meet the inclusion criteria, leaving 76 studies that were analysed in this systematic review.

Of the 76 studies, 35 investigated the risk of CVM with respect to RT laterality, ${ }^{5-39} 28$ studies investigated the risk of CVEs with respect to RT laterality, 7,10-12,16,18,20,22,24,33,40-57 26 studies investigated the risk of CVM with respect to RT compared with no RT, $, 7,10,14,21,27,29,30,32,38,54,58-72$ and 22 studies investigated the risk of CVEs with respect

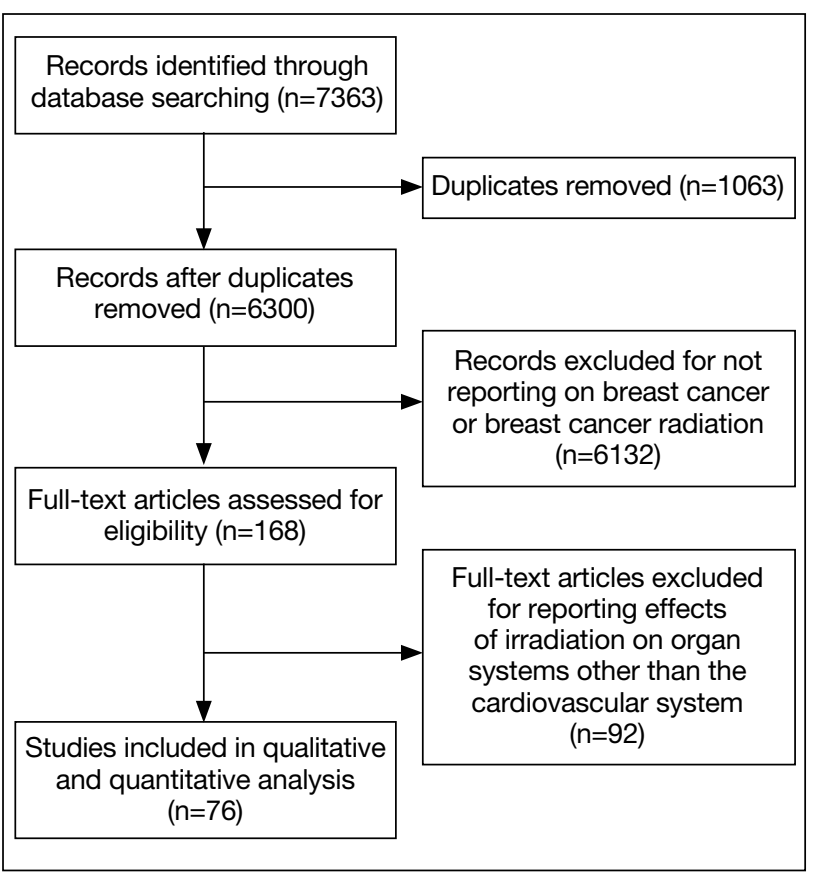

Figure. PRISMA flow diagram. to RT compared with no RT. $7,36,44,45,48,49,54,59,61-63,67-69,73-80$ Several studies overlapped between the categories and investigated the risk of CVEs and/or CVM with respect to RT and/or RT laterality. Results from the largest studies, based on study population size, will be highlighted for each of the four categories. The results for all studies are also reported in Tables 1 to 4 .

\section{Cardiovascular Mortality in Patients with Left-sided or Right-sided Radiotherapy}

Of 35 studies investigating the risk of CVM with respect to RT laterality, seven (20\%) found a significant increased risk of CVM in patients who received leftsided RT compared with patients who received rightsided RT, all of which included study periods that started prior to 1985 (Table 1). ${ }^{8,10,21,26,28,30,39}$ An additional six studies found a significant association between laterality and CVM only in subgroup analysis. ${ }^{5,11,23,32,34,36}$ Of these six studies, three had subgroup analyses based on time period stratification, where in general older study periods before 1980-1990 were significant while more recent time periods were not (Table 1). .,23,34 $^{2}$

\section{Cardiovascular Events in Patients with Left-sided Radiotherapy or Right-sided Radiotherapy}

Of 28 studies investigating the risk of CVEs with respect to RT laterality, eight (29\%) found a significant increased risk of CVEs in patients who received left-sided RT compared with patients who received right-sided RT (Table 2). ${ }^{11,18,40,41,46,49,51,56}$ Of these eight studies, five included study periods that started prior to $1985 .{ }^{11,18,40,41,46}$ An additional three studies found a significant association between laterality and CVEs only in subgroup analysis. ${ }^{22,47,55}$ These subgroup analyses were based on different treatment types and differences in types of CVEs.

\section{Cardiovascular Mortality in Patients with Radiotherapy or without Radiotherapy}

Of 26 studies investigating the risk of CVM for patients that received RT compared with those who did not receive RT, seven (27\%) found a significant increase in CVM (Table 3)..$^{29,30,32,63,64,71,72}$ Of these seven studies, six included study periods that started prior to 1985 . An additional five studies found a significant association between RT and CVM only in subgroup analysis. . $10,21,68,70$ These subgroup analyses were based on different treatment types and differences in specific causes of CVM, such as death from cardiac diseases compared with death from vascular diseases. 
P Taylor, S Chan, AB Wan, et al

Table 1. Studies assessing the risk of cardiovascular mortality in patients who received left-sided RT compared with those who received right-sided RT.

\begin{tabular}{|c|c|c|c|c|c|c|c|c|}
\hline $\begin{array}{l}\text { Study (first } \\
\text { author) }\end{array}$ & Study population & Country & $\begin{array}{l}\text { Treat- } \\
\text { ment } \\
\text { period }\end{array}$ & Study design & $\begin{array}{l}\text { Median or } \\
\text { mean follow- } \\
\text { up time, y }\end{array}$ & Endpoint(s) & Endpoint criteria & $\begin{array}{l}\text { Significant increased risk } \\
\text { of cardiovascular death }\end{array}$ \\
\hline $\begin{array}{l}\text { Henson } \\
(2020)^{5}\end{array}$ & $1,018,505$ & Multinational & $\begin{array}{l}1987- \\
2002\end{array}$ & $\begin{array}{l}\text { Population- } \\
\text { based cohort }\end{array}$ & 6.7 & Cardiac mortality & Did not specify & $\begin{array}{l}\text { Yes for women diagnosed } \\
\text { before } 1990(\mathrm{RR}=1.134 \text {, } \\
95 \% \mathrm{Cl}=1.09-1.17) \text {, but } \\
\text { not for women diagnosed } \\
\text { after } 1990 \text { (RR=0.98, } \\
95 \% \mathrm{Cl}=0.93-1.02)\end{array}$ \\
\hline Beaton (2019) ${ }^{6}$ & 5249 & Canada & $\begin{array}{l}2002- \\
2006\end{array}$ & $\begin{array}{l}\text { Population- } \\
\text { based } \\
\text { case-control }\end{array}$ & $\begin{array}{l}\text { 10-year } \\
\text { cardiac } \\
\text { mortality }\end{array}$ & $\begin{array}{l}\text { Cardiovascular } \\
\text { deaths (CAD, } \\
\text { Ml, CHF, } \\
\text { cardiomyopathy, } \\
\text { arrhythmias, } \\
\text { pericardial disease } \\
\text { and 'other' heart } \\
\text { disease) }\end{array}$ & ICD-10 & $\begin{array}{l}\text { No }(1.7 \% \text { for left-sided vs. } \\
1.3 \% \text { for right-sided, log } \\
\text { rank } p=0.30) \\
\text { No for CAD }(p=0.26) \text {, } \\
\text { cardiac arrest ( } p=0.34) \text {, } \\
\text { CHF and cardiomyopathy } \\
\text { (0.75), conduction } \\
\text { disorders and arrhythmias } \\
(p=0.66), \text { valvular heart } \\
\text { disease }(0.1) \text {, or other } \\
\text { heart diseases }(p=0.18)\end{array}$ \\
\hline Li (2018) & 168,761 & $\begin{array}{l}\text { United } \\
\text { States }\end{array}$ & $\begin{array}{l}2000- \\
2008\end{array}$ & $\begin{array}{l}\text { Population- } \\
\text { based cohort }\end{array}$ & 8.8 & Cardiac mortality & Did not specify & $\begin{array}{l}\text { No (right-sided vs. left- } \\
\text { sided } \mathrm{RT}, \mathrm{HR}=1.025 \\
95 \% \mathrm{Cl}=0.856-1.099 \\
\mathrm{p}=0.481 \text { ) }\end{array}$ \\
\hline Obi $(2018)^{27}$ & 2439 & Germany & $\begin{array}{l}2001- \\
2005\end{array}$ & $\begin{array}{l}\text { Population- } \\
\text { based } \\
\text { case-control }\end{array}$ & 11.9 & Cardiac mortality & ICD-10 & $\begin{array}{l}\text { No }(H R=0.96 \\
95 \% \mathrm{Cl}=0.54-1.71)\end{array}$ \\
\hline $\begin{array}{l}\text { Chang } \\
(2017)^{33}\end{array}$ & $\begin{array}{l}2577 \text { for } 1 \\
\text { institution which } \\
\text { was used for } \\
\text { acute coronary } \\
\text { event (YCCR) } \\
\text { and } 24,235 \text { for } \\
\text { nationwide registry } \\
\text { analysis used for } \\
\text { cardiac mortality } \\
\text { (KBCR) }\end{array}$ & Korea & $\begin{array}{l}1990- \\
2012\end{array}$ & $\begin{array}{l}\text { Population- } \\
\text { based cohort }\end{array}$ & $\begin{array}{l}7 \text { for } Y C C R \\
\text { and } 7.9 \text { for } \\
\text { KBCR }\end{array}$ & $\begin{array}{l}\text { Acute coronary } \\
\text { event (death } \\
\text { resulting from heart } \\
\text { disease, or newly } \\
\text { diagnosed IHD) }\end{array}$ & ICD-10 & $\begin{array}{l}\text { No in the KBCR }(H R=1.52 \text {, } \\
95 \% C l=0.37-6.25) \text { and } \\
\text { YCCR }(p=0.347)\end{array}$ \\
\hline $\begin{array}{l}\text { Haque } \\
(2017)^{34}\end{array}$ & 140,914 & $\begin{array}{l}\text { United } \\
\text { States }\end{array}$ & $\begin{array}{l}1973- \\
2002\end{array}$ & $\begin{array}{l}\text { Population- } \\
\text { based cohort }\end{array}$ & 11.5 & Cardiac mortality & Not specified & $\begin{array}{l}\text { Yes from } 1973-1982 \\
(\mathrm{HR}=1.295 \text {, } \\
95 \% \mathrm{Cl}=1.182-1.420) \\
\mathrm{No} \text { from } 1983-1992 \\
(\mathrm{HR}=1.022 \text {, } \\
95 \% \mathrm{Cl}=0.949-1.100) \text { or } \\
1993-2002(\mathrm{HR}=0.989 \text {, } \\
95 \% \mathrm{Cl}=0.935-1.046)\end{array}$ \\
\hline $\begin{array}{l}\text { Merzenich } \\
(2017)^{35}\end{array}$ & 9058 & Germany & $\begin{array}{l}1998- \\
2008\end{array}$ & $\begin{array}{l}\text { Multicentre } \\
\text { cohort }\end{array}$ & 6.5 & $\begin{array}{l}\text { Death due to } \\
\text { MI, chronic IHD, } \\
\text { acute IHD, CHF, } \\
\text { angina, cardiac } \\
\text { arrest, conduction } \\
\text { disorder or vitium } \\
\text { cordis }\end{array}$ & ICD-10 & $\begin{array}{l}\text { No (HR=0.94, } \\
95 \% \mathrm{Cl}=0.64-1.38)\end{array}$ \\
\hline $\begin{array}{l}\text { Boekel } \\
(2016)^{36}\end{array}$ & 27,380 & Netherlands & $\begin{array}{l}1989- \\
2005\end{array}$ & $\begin{array}{l}\text { Population- } \\
\text { based cohort }\end{array}$ & 9 & $\begin{array}{l}\text { Cardiovascular } \\
\text { event (a } \\
\text { cardiovascular } \\
\text { hospital discharge } \\
\text { diagnosis, } \\
\text { cardiosurgical } \\
\text { intervention, or } \\
\text { death due to CVD) }\end{array}$ & ICD-10 & $\begin{array}{l}\text { Yes for } \mathrm{RT} \text { after } \\
\text { mastectomy }(\mathrm{HR}=1.19 \text {, } \\
95 \% \mathrm{Cl}=1.04-1.36) \\
\text { No for } \mathrm{RT} \text { after WLE } \\
(\mathrm{HR}=1.07, \\
95 \% \mathrm{Cl}=0.98-1.16)\end{array}$ \\
\hline $\begin{array}{l}\text { Paul Wright } \\
(2016)^{37}\end{array}$ & 66,687 & $\begin{array}{l}\text { United } \\
\text { States }\end{array}$ & $\begin{array}{l}1990- \\
1999\end{array}$ & $\begin{array}{l}\text { Population- } \\
\text { based cohort }\end{array}$ & 15.5 & Cardiac mortality & $\begin{array}{l}\text { SEER cause- } \\
\text { specific death } \\
\text { classification }\end{array}$ & $\begin{array}{l}\text { No difference in } 5-\text {, } \\
10 \text { - and } 15 \text {-year cardiac } \\
\text { mortality }(p=0.435)\end{array}$ \\
\hline Ye $(2015)^{38}$ & 2796 & $\begin{array}{l}\text { United } \\
\text { States }\end{array}$ & $\begin{array}{l}1990- \\
1997\end{array}$ & $\begin{array}{l}\text { Population- } \\
\text { based cohort }\end{array}$ & 15 & Cardiac mortality & $\begin{array}{l}\text { Cause of death } \\
\text { codes }\end{array}$ & $\begin{array}{l}\text { No. There was a lower } \\
\text { cardiac mortality rate for } \\
\text { left-sided RT than for right- } \\
\text { sided RT ( } 4.6 \% \text { vs. } 6.0 \% \text {, } \\
\text { respectively; } p=0.04 \text { ) }\end{array}$ \\
\hline
\end{tabular}

Abbreviations: $95 \% \mathrm{Cl}$ = 95\% confidence interval; $\mathrm{AMI}=$ acute myocardial infarction; $\mathrm{CAD}=$ coronary artery disease; $\mathrm{CHF}=$ congestive heart failure; CVD = cardiovascular disease; $\mathrm{DCIS}=$ ductal carcinoma in situ; $\mathrm{HR}=$ hazard ratio; ICD = International Classification of Diseases; $\mathrm{IHD}$ = ischaemic heart disease; $\mathrm{KBCR}=$ Korean Breast Cancer Society; $\mathrm{Ml}=$ myocardial infarction; $\mathrm{N} / \mathrm{A}=$ not available; $\mathrm{RCT}=$ randomised controlled trial; RR = risk ratio; RT = radiotherapy; SEER = Surveillance, Epidemiology, and End Results Program; SMR = standardised mortality ratio; $\mathrm{WLE}=$ wide local excision; YCCR = Yonsei Cancer Center. 
Table 1. (cont'd)

\begin{tabular}{|c|c|c|c|c|c|c|c|c|}
\hline $\begin{array}{l}\text { Study (first } \\
\text { author) }\end{array}$ & Study population & Country & $\begin{array}{l}\text { Treat- } \\
\text { ment } \\
\text { period }\end{array}$ & Study design & $\begin{array}{l}\text { Median or } \\
\text { mean follow- } \\
\text { up time, y }\end{array}$ & Endpoint(s) & Endpoint criteria & $\begin{array}{l}\text { Significant increased risk } \\
\text { of cardiovascular death }\end{array}$ \\
\hline $\begin{array}{l}\text { Boekel } \\
(2014)^{7}\end{array}$ & $\begin{array}{l}2899 \text { (DCIS } \\
\text { patients only) }\end{array}$ & Netherlands & $\begin{array}{l}1989- \\
2004\end{array}$ & $\begin{array}{l}\text { Population- } \\
\text { based cohort }\end{array}$ & 10 & $\begin{array}{l}\text { Death due to MI, } \\
\text { other IHDs, other } \\
\text { heart disease, } \\
\text { pericarditis, } \\
\text { valvular } \\
\text { dysfunction, } \\
\text { cardiomyopathy, } \\
\text { arrhythmia, } \\
\text { CHF, and/or } \\
\text { cerebrovascular } \\
\text { disease }\end{array}$ & ICD-10 & $\begin{array}{l}\text { No }(H R=0.70 \\
95 \% \mathrm{Cl}=0.31-1.56)\end{array}$ \\
\hline $\begin{array}{l}\text { Henson } \\
(2013)^{8}\end{array}$ & 256,976 & $\begin{array}{l}\text { United } \\
\text { States }\end{array}$ & $\begin{array}{l}1973- \\
2008\end{array}$ & $\begin{array}{l}\text { Population- } \\
\text { based cohort }\end{array}$ & $\begin{array}{l}\text { Not specified. } \\
\text { Studied up to } \\
20+\text { years of } \\
\text { follow-up }\end{array}$ & $\begin{array}{l}\text { Death due to heart } \\
\text { disease }\end{array}$ & ICD-10 & $\begin{array}{l}\text { Overall, yes }(\mathrm{HR}=1.08, \\
95 \% \mathrm{Cl}=1.03-1.14 ; \\
2 \mathrm{p}=0.002) \\
\text { Yes from } 1973-1982 \\
<10 \text { years after diagnosis } \\
(\mathrm{HR}=1.19,95 \% \mathrm{Cl}= \\
1.02-1.38), 10-14 \text { years } \\
\text { after diagnosis }(\mathrm{HR}=1.35, \\
95 \% \mathrm{Cl}=1.05-1.73), 15-19 \\
\text { years after diagnosis } \\
(\mathrm{HR}=1.64,95 \% \mathrm{Cl}= \\
1.26-2.14) \text { and } 20+\text { years } \\
\text { after diagnosis }(\mathrm{HR}=1.90, \\
95 \% \mathrm{Cl}=1.52-2.37) \\
\mathrm{No} \text { after } 1982\end{array}$ \\
\hline $\begin{array}{l}\text { Tjessem } \\
(2013)^{9}\end{array}$ & $\begin{array}{l}1107 \text { in } 4.3-\text {-Gy } \\
\text { group and } 459 \text { in } \\
2.5 \text {-Gy group }\end{array}$ & Norway & $\begin{array}{l}1975- \\
1991\end{array}$ & $\begin{array}{l}\text { Population- } \\
\text { based } \\
\text { case-control }\end{array}$ & 20 & $\begin{array}{l}\text { Cardiovascular } \\
\text { death (death due } \\
\text { to IHD or CVD) }\end{array}$ & $\begin{array}{l}\text { ICD-9 and } \\
\text { ICD-10 }\end{array}$ & $\begin{array}{l}\text { No }(H R=0.93 \\
95 \% \mathrm{Cl}=0.52-1.66 \\
p=0.812)\end{array}$ \\
\hline $\begin{array}{l}\text { Bouillon } \\
(2011)^{10}\end{array}$ & 3038 & France & $\begin{array}{l}1954- \\
1984\end{array}$ & $\begin{array}{l}\text { Single-centre } \\
\text { cohort }\end{array}$ & 28 & $\begin{array}{l}\text { Death due to } \\
\text { cardiac diseases } \\
\text { (pericarditis, } \\
\text { myocarditis, } \\
\text { valvular heart } \\
\text { diseases, IHD, } \\
\text { heart failure) }\end{array}$ & $\begin{array}{l}\text { ICD-8, ICD-9, } \\
\text { ICD-10 }\end{array}$ & $\begin{array}{l}\text { Yes, 1.56-fold } \\
\text { (95\% } \mathrm{Cl}=1.27-1.90) \text { higher } \\
\text { risk of dying of cardiac } \\
\text { disease }\end{array}$ \\
\hline $\begin{array}{l}\text { McGale } \\
(2011)^{11}\end{array}$ & 34,825 & $\begin{array}{l}\text { Sweden } \\
\text { and } \\
\text { Denmark }\end{array}$ & $\begin{array}{l}1976- \\
2006\end{array}$ & $\begin{array}{l}\text { Population- } \\
\text { based cohort }\end{array}$ & Not specified & $\begin{array}{l}\text { Death due to heart } \\
\text { disease }\end{array}$ & $\begin{array}{l}\text { ICD-8, ICD-9, } \\
\text { ICD-10 }\end{array}$ & $\begin{array}{l}\text { Overall, no for all IHDs } \\
(\mathrm{HR}=1.00,95 \% \mathrm{Cl}= \\
0.86-1.15) \text { or for heart } \\
\text { disease other than } \mathrm{IHD} \\
(\mathrm{HR}=1.00,95 \% \mathrm{Cl}= \\
0.81-1.22) \\
\text { Yes for death due to } \mathrm{AMl} \\
(\mathrm{HR}=1.23,95 \% \mathrm{Cl}= \\
1.01-1.49 ; \mathrm{p}=0.04)\end{array}$ \\
\hline Park $(2011)^{12}$ & $\begin{array}{l}129 \text { (DCIS patients } \\
\text { only) }\end{array}$ & $\begin{array}{l}\text { United } \\
\text { States }\end{array}$ & $\begin{array}{l}1986- \\
2002\end{array}$ & $\begin{array}{l}\text { Single-centre } \\
\text { cohort }\end{array}$ & 8.2 & $\begin{array}{l}\text { Death due to } \\
\text { CAD, MI, CHF, } \\
\text { chronic IHD, } \\
\text { arrhythmia, valvular } \\
\text { disease, and } \\
\text { cardiomyopathy }\end{array}$ & Did not specify & No $(p=0.64)$ \\
\hline $\begin{array}{l}\text { Stokes } \\
(2011)^{13}\end{array}$ & 4929 & Canada & $\begin{array}{l}1990- \\
1996\end{array}$ & $\begin{array}{l}\text { Population- } \\
\text { based cohort }\end{array}$ & 11.7 & $\begin{array}{l}\text { Cardiac and } \\
\text { cerebrovascular } \\
\text { deaths }\end{array}$ & Did not specify & $\begin{array}{l}\text { No }(R R=1.02 \\
95 \% \mathrm{Cl}=0.77-1.35 \\
\mathrm{p}=0.89)\end{array}$ \\
\hline Wang $(2011)^{14}$ & 519 & Australia & $\begin{array}{l}1 \text { Apr- } \\
30 \\
\text { Sep } \\
1995\end{array}$ & $\begin{array}{l}\text { Population- } \\
\text { based cohort }\end{array}$ & Not specified & $\begin{array}{l}\text { 10-year cardiac } \\
\text { mortality }\end{array}$ & $\begin{array}{l}\text { Death certificates } \\
\text { codes by the } \\
\text { Australian } \\
\text { Bureau of } \\
\text { Statistics }\end{array}$ & No $(p=0.63)$ \\
\hline $\begin{array}{l}\text { Bouchardy } \\
(2010)^{15}\end{array}$ & 1245 & Switzerland & $\begin{array}{l}1980- \\
2004\end{array}$ & $\begin{array}{l}\text { Population- } \\
\text { based cohort }\end{array}$ & 7.7 & $\begin{array}{l}\text { Cardiovascular } \\
\text { mortality }\end{array}$ & ICD-10 & $\begin{array}{l}\text { No }(\mathrm{HR}=0.5 \\
95 \% \mathrm{Cl}=0.2-1.1)\end{array}$ \\
\hline Gutt $(2008)^{16}$ & 41 & $\begin{array}{l}\text { United } \\
\text { States }\end{array}$ & $\begin{array}{l}1980- \\
1994\end{array}$ & $\begin{array}{l}\text { Single-centre } \\
\text { cohort }\end{array}$ & $\begin{array}{l}7.9 \text { for left- } \\
\text { sided, } 11.3 \text { for } \\
\text { right-sided }\end{array}$ & $\begin{array}{l}\text { Cardiovascular } \\
\text { mortality following } \\
\text { MI, CHF and/or } \\
\text { CAD }\end{array}$ & Not specified & No $(H R=4.2 ; p=0.08)$ \\
\hline $\begin{array}{l}\text { Borger } \\
(2007)^{18}\end{array}$ & 1601 & Netherlands & $\begin{array}{l}1980- \\
1993\end{array}$ & $\begin{array}{l}\text { Multicentre } \\
\text { cohort }\end{array}$ & 16 & $\begin{array}{l}\text { CVD (including IHD } \\
\text { and other heart } \\
\text { diseases) }\end{array}$ & ICD-9 & $\begin{array}{l}\text { No for CVD }(\mathrm{HR}=1.57 \text {, } \\
95 \% \mathrm{Cl}=0.83-3.00) \text { and } \\
\mathrm{IHD}(\mathrm{HR}=1.99 \text {, } \\
95 \% \mathrm{Cl}=0.71-5.59)\end{array}$ \\
\hline
\end{tabular}


Table 1. (cont'd)

\begin{tabular}{|c|c|c|c|c|c|c|c|c|}
\hline $\begin{array}{l}\text { Study (first } \\
\text { author) }\end{array}$ & Study population & Country & $\begin{array}{l}\text { Treat- } \\
\text { ment } \\
\text { period }\end{array}$ & Study design & $\begin{array}{l}\text { Median or } \\
\text { mean follow- } \\
\text { up time, y }\end{array}$ & Endpoint(s) & Endpoint criteria & $\begin{array}{l}\text { Significant increased risk } \\
\text { of cardiovascular death }\end{array}$ \\
\hline $\begin{array}{l}\text { Marhin } \\
(2007)^{19}\end{array}$ & 7477 & Canada & $\begin{array}{l}1984- \\
2000\end{array}$ & $\begin{array}{l}\text { Population- } \\
\text { based cohort }\end{array}$ & 7.9 & $\begin{array}{l}\text { Death due to } \\
\text { AMI, aortic } \\
\text { stenosis, CHF, } \\
\text { atherosclerotic } \\
\text { CVD, } \\
\text { atherosclerotic } \\
\text { heart disease, } \\
\text { arrhythmia, } \\
\text { cardiac arrest, } \\
\text { CVD, IHD, chronic } \\
\text { IHD, coronary } \\
\text { atherosclerosis, } \\
\text { endocarditis, or } \\
\text { essential primary } \\
\text { hypertension }\end{array}$ & $\begin{array}{l}\text { Oncology } \\
\text { Reporting } \\
\text { System } \\
\text { database death } \\
\text { certificates }\end{array}$ & No $(p=0.69)$ \\
\hline $\begin{array}{l}\text { Paszat } \\
(2007)^{20}\end{array}$ & 619 & Canada & $\begin{array}{l}1982- \\
1988\end{array}$ & $\begin{array}{l}\text { Population- } \\
\text { based cohort }\end{array}$ & 13.5 minimum & $\begin{array}{l}\mathrm{AMl} \text { and death due } \\
\text { to } \mathrm{AMI}\end{array}$ & Chart review & $\begin{array}{l}\text { No }(H R=1.07 \\
95 \% \mathrm{Cl}=0.65-1.72)\end{array}$ \\
\hline $\begin{array}{l}\text { Roychoudhuri } \\
(2007)^{21}\end{array}$ & 20,871 & $\begin{array}{l}\text { South East } \\
\text { England }\end{array}$ & $\begin{array}{l}1971- \\
1988\end{array}$ & $\begin{array}{l}\text { Population- } \\
\text { based cohort }\end{array}$ & 18.5 & $\begin{array}{l}\text { Death due to IHD } \\
\text { and other CVDs }\end{array}$ & ICD-9, 10 & $\begin{array}{l}\text { Yes for overall CVD } \\
\text { mortality }(\mathrm{HR}=1.25 \text {, } \\
95 \% \mathrm{Cl}=1.05-1.49 \\
\mathrm{p}=0.014) \\
\text { No for } \mathrm{HD} \text { mortality } \\
\text { specifically }(\mathrm{HR}=1.23 \text {, } \\
95 \% \mathrm{Cl}=0.95-1.60 ; \\
\mathrm{p}=0.114)\end{array}$ \\
\hline Harris $(2006)^{22}$ & 961 & $\begin{array}{l}\text { United } \\
\text { States }\end{array}$ & $\begin{array}{l}1977- \\
1994\end{array}$ & $\begin{array}{l}\text { Single-centre } \\
\text { cohort }\end{array}$ & 12 & $\begin{array}{l}\text { Freedom from } \\
\text { cardiac death } \\
\text { (death due to } \mathrm{Ml} \text { or } \\
\mathrm{CHF} \text { ) at } 5,10,15 \text {, } \\
\text { and } 20 \text { years }\end{array}$ & Not specified & $\begin{array}{l}\text { No for cardiac death } \\
\text { overall }(p=0.25) \\
\text { No for } M I(p=0.22) \text { and } \\
\text { CHF }(p=0.82)\end{array}$ \\
\hline Darby $(2005)^{39}$ & 115,165 & $\begin{array}{l}\text { United } \\
\text { States }\end{array}$ & $\begin{array}{l}1973- \\
2001\end{array}$ & $\begin{array}{l}\text { Population- } \\
\text { based cohort }\end{array}$ & $\mathrm{N} / \mathrm{A}$ & $\begin{array}{l}\text { 5-, } 10-, \text { and } 15- \\
\text { year death rates } \\
\text { due to AMI, IHD } \\
\text { and other heart } \\
\text { disease }\end{array}$ & ICD-9 & $\begin{array}{l}\text { Overall, yes }(\mathrm{HR}=1.16, \\
95 \% \mathrm{Cl}=1.08-1.24 ; \\
2 \mathrm{p}=0.00004) \\
\text { Yes for patients diagnosed } \\
\text { during } 1973-1982 \\
<10 \text { years after diagnosis } \\
\text { (HR=1.20, } 95 \% \mathrm{Cl}= \\
1.04-1.38), 10-14 \text { years } \\
\text { after diagnosis }(\mathrm{HR}=1.42 \text {, } \\
95 \% \mathrm{Cl}=1.11-1.82) \text { and } \\
\geq 15 \text { years after diagnosis } \\
(\mathrm{HR}=1.58,95 \% \mathrm{Cl}= \\
1.29-1.95) \\
\mathrm{No}, \text { during } 1983-1992 \\
<10 \text { years after diagnosis } \\
(\mathrm{HR}=1.04,95 \% \mathrm{Cl}= \\
0.91-1.18) \text { or }>10 \text { years } \\
\text { after diagnosis }(\mathrm{HR}=1.27, \\
95 \% \mathrm{Cl}=0.99-1.63) \\
\text { or during } 1993-2001 \\
(\mathrm{HR}=0.96,95 \% \mathrm{Cl}= \\
0.82-1.12)\end{array}$ \\
\hline $\begin{array}{l}\text { Giordano } \\
(2005)^{23}\end{array}$ & 27,283 & $\begin{array}{l}\text { United } \\
\text { States }\end{array}$ & $\begin{array}{l}1973- \\
1989\end{array}$ & $\begin{array}{l}\text { Population- } \\
\text { based cohort }\end{array}$ & $\begin{array}{l}10.1(1973- \\
1979), 11.1 \\
(1980-1984), \\
\text { and 11 (1985- } \\
\text { 1989) }\end{array}$ & Death due to IHD & ICD-9, ICD-10 & $\begin{array}{l}\text { Overall, } n o(p=0.07) \\
\text { Yes for patients diagnosed } \\
\text { between } 1973 \text { and } 1979 \\
(p=0.02) \text {. No between } \\
1980 \text { and } 1984(p=0.64) \\
\text { and } 1985-1989(p=0.98)\end{array}$ \\
\hline Vallis $(2002)^{24}$ & 2128 & Canada & $\begin{array}{l}1982- \\
1988\end{array}$ & $\begin{array}{l}\text { Single-centre } \\
\text { cohort }\end{array}$ & 10.2 & Death due to $\mathrm{Ml}$ & ICD-9 & No \\
\hline Nixon $(1998)^{25}$ & 745 & $\begin{array}{l}\text { United } \\
\text { States }\end{array}$ & $\begin{array}{l}1968- \\
1986\end{array}$ & $\begin{array}{l}\text { Single-centre } \\
\text { cohort }\end{array}$ & $\begin{array}{l}\text { Not specified. } \\
\text { Studied } 12 \\
\text { years of } \\
\text { follow-up }\end{array}$ & Cardiac mortality & Not specified & No $(R R=1.3 ; p=0.29)$ \\
\hline $\begin{array}{l}\text { Paszat } \\
(1999)^{26}\end{array}$ & 3006 & Canada & $\begin{array}{l}1982- \\
1987\end{array}$ & $\begin{array}{l}\text { Population- } \\
\text { based cohort }\end{array}$ & 8.8 & Death due to Ml & ICD-9 & $\begin{array}{l}\text { Yes }(\mathrm{RR}=2.10 \\
95 \% \mathrm{Cl}=1.11-3.95)\end{array}$ \\
\hline $\begin{array}{l}\text { Paszat } \\
(1998)^{28}\end{array}$ & 47,948 & $\begin{array}{l}\text { United } \\
\text { States }\end{array}$ & $\begin{array}{l}1973- \\
1992\end{array}$ & $\begin{array}{l}\text { Population- } \\
\text { based cohort }\end{array}$ & $74 \mathrm{mo}$ & Death due to Ml & ICD-9 & $\begin{array}{l}\text { Yes }(\mathrm{RR}=1.17 \\
95 \% \mathrm{Cl}=1.01-1.36)\end{array}$ \\
\hline $\begin{array}{l}\text { Cuzick } \\
(1994)^{29}\end{array}$ & 3970 & Multinational & $\begin{array}{l}1949- \\
1974\end{array}$ & Multiple RCTs & $>10$ & Cardiac mortality & ICD-9 & No (SMR=1.34; $p=0.9)$ \\
\hline
\end{tabular}


Table 1. (cont'd)

\begin{tabular}{|c|c|c|c|c|c|c|c|c|}
\hline $\begin{array}{l}\text { Study (first } \\
\text { author) }\end{array}$ & Study population & Country & $\begin{array}{l}\text { Treat- } \\
\text { ment } \\
\text { period }\end{array}$ & Study design & $\begin{array}{l}\text { Median or } \\
\text { mean follow- } \\
\text { up time, y }\end{array}$ & Endpoint(s) & Endpoint criteria & $\begin{array}{l}\text { Significant increased risk } \\
\text { of cardiovascular death }\end{array}$ \\
\hline $\begin{array}{l}\text { Houghton } \\
(1994)^{30}\end{array}$ & 1376 & $\begin{array}{l}\text { United } \\
\text { Kingdom }\end{array}$ & $\begin{array}{l}1970- \\
1975\end{array}$ & RCT & 19 & $\begin{array}{l}\text { Cardiac death } \\
\text { (death due to heart } \\
\text { failure, myocardial } \\
\text { infarct, or coronary } \\
\text { thrombosis) }\end{array}$ & Did not specify & Yes $(\times 2($ int $)=5.08 ; p=0.02)$ \\
\hline $\begin{array}{l}\text { Rutqvist } \\
(1990)^{31}\end{array}$ & 54,617 & Sweden & $\begin{array}{l}1970- \\
1985\end{array}$ & $\begin{array}{l}\text { Population- } \\
\text { based cohort }\end{array}$ & 9 & $\begin{array}{l}\text { Deaths due to } \\
\text { CVDs and Mls }\end{array}$ & Did not specify & $\begin{array}{l}\text { No for overall CVD } \\
\text { mortality (RR=1.03, } \\
95 \% \mathrm{Cl}=0.98-1.09 \text { ) } \\
\text { Yes for Ml mortality } \\
\text { (RR=1.09, 95\% Cl= } \\
1.02-1.17 \text { ) }\end{array}$ \\
\hline $\begin{array}{l}\text { Haybittle } \\
(1989)^{32}\end{array}$ & 1376 & $\begin{array}{l}\text { United } \\
\text { Kingdom }\end{array}$ & $\begin{array}{l}1970- \\
1975\end{array}$ & RCT & $13.2-18$ & $\begin{array}{l}\text { Cardiac deaths } \\
\text { (deaths due to } \\
\text { heart failure, } \\
\text { Ml, coronary } \\
\text { thrombosis, } \\
\text { and other } \\
\text { cardiovascular } \\
\text { deaths) }\end{array}$ & $\begin{array}{l}\text { Death report } \\
\text { forms, copies } \\
\text { of death } \\
\text { certificates, and } \\
\text { correspondence } \\
\text { with treating } \\
\text { clinicians and } \\
\text { examination of } \\
\text { the patient's } \\
\text { notes }\end{array}$ & $\begin{array}{l}\text { No overall } \\
\text { Yes for left-sided RT vs. } \\
\text { no left-sided RT (RR=2.26, } \\
95 \% \mathrm{Cl}=1.19-4.29) \text { and no } \\
\text { for right-sided } \mathrm{RT} \text { vs. no } \\
\text { right-sided } \mathrm{RT} \text { (RR=1.20, } \\
95 \% \mathrm{Cl}=0.64-2.28) \\
\text { Interaction test between } \\
\text { left and right is insignificant } \\
(\mathrm{p}=0.17)\end{array}$ \\
\hline
\end{tabular}

\section{Cardiovascular Events in Patients with Radiotherapy or without Radiotherapy}

Of 22 studies investigating the risk of CVEs for patients that received RT compared with those who did not receive RT, five $(23 \%)$ found a significant association between RT and CVEs (Table 4). ${ }^{36,73,75,79,80}$ An additional three studies found a significant association between RT and CVEs only in subgroup analysis. ${ }^{74,77,78}$ These subgroup analyses were based on different treatment types and the presence of pre-existing cardiovascular risk factors.

\section{DISCUSSION}

This review summarises the cardiovascular morbidity and mortality risk associated with breast adjuvant RT and the laterality of the RT. When comparing patients who received left-sided $\mathrm{RT}$ with those who received right-sided RT, we found that 7 of 35 studies found a significant increase in the risk of CVM and 8 of 28 studies found a significantly increased risk of CVEs. For patients who received RT compared with those who did not receive RT, 7 of 26 studies found a significantly increased risk of CVM and 5 of 22 studies found a significant increased risk of CVEs.

A previous meta-analysis conducted by Cheng et $\mathrm{al}^{4}$ examined studies of breast cancer patients from 1966 to 2015 . The authors ${ }^{4}$ found that patients who received RT had an increased risk of coronary heart disease ( $\mathrm{RR}=1.30,95 \% \mathrm{CI}=1.13-1.49)$ and cardiac mortality ( $R R=1.38,95 \% \mathrm{CI}=1.18-1.62)$ compared with patients who did not receive RT. They also found that patients who received left-sided RT experienced an increased risk of developing coronary heart disease compared with patients receiving right-sided $\mathrm{RT}(\mathrm{RR}=1.29$, 95\% CI=1.13-1.48). ${ }^{4}$ Patients receiving left-sided $\mathrm{RT}$ also experienced an increased risk of cardiac death compared with patients receiving right-sided $\mathrm{RT}(\mathrm{RR}=1.22$, 95\% $\mathrm{CI}=1.08-1.37) .{ }^{4}$ In contrast to Cheng et $\mathrm{al},{ }^{4}$ in the present review, we found newer studies in which there was no significant increased risk of CVEs or CVM in patients who received RT compared with patients who did not receive $\mathrm{RT}$. We also found more studies in which patients who received left-sided RT had no significant increased risk of CVEs or CVM compared with patients who received right-sided $\mathrm{RT}$. These differences likely reflect the fact that the present review includes many new studies since 2015 in which the study populations received modern RT techniques. However, because we did not conduct a meta-analysis in the present review, it remains unclear whether our findings represent a significantly different association between breast cancer RT and cardiovascular risk compared with that reported by Cheng et al. ${ }^{4}$ 
P Taylor, S Chan, AB Wan, et al

Table 2. Studies assessing the risk of cardiovascular events in patients who received left-sided RT compared with those who received right-sided RT.

\begin{tabular}{|c|c|c|c|c|c|c|c|c|}
\hline $\begin{array}{l}\text { Study (first } \\
\text { author) }\end{array}$ & Study population & Country & $\begin{array}{l}\text { Treatment } \\
\text { period }\end{array}$ & Study design & $\begin{array}{l}\text { Median or } \\
\text { mean follow- } \\
\text { up time, y }\end{array}$ & Endpoint(s) & Endpoint criteria & $\begin{array}{l}\text { Significant increased risk } \\
\text { of cardiovascular death }\end{array}$ \\
\hline $\begin{array}{l}\text { Killander } \\
(2020)^{54}\end{array}$ & 563 & Sweden & $\begin{array}{l}1991- \\
1997\end{array}$ & $\mathrm{RCT}$ & 21.3 & $\begin{array}{l}\text { Cardiac morbidity } \\
\text { and mortality }\end{array}$ & $\begin{array}{l}\text { ICD-8, ICD-9, } \\
\text { ICD-10 }\end{array}$ & $\begin{array}{l}\text { No for } \mathrm{PCl}(3.8 \% \text { for } \\
\text { right-sided, } 2.2 \% \text { for } \\
\text { left-sided, } \mathrm{p}=0.33 \text { ) } \\
\text { No for open heart surgery } \\
\text { (5.2\% for right-sided, } \\
3.4 \% \text { for left-sided, } \\
p=0.06 \text { ) }\end{array}$ \\
\hline $\begin{array}{l}\text { Wennstig } \\
(2020)^{56}\end{array}$ & 37,427 & Sweden & $\begin{array}{l}1992- \\
2012\end{array}$ & $\begin{array}{l}\text { Population- } \\
\text { based cohort }\end{array}$ & 8.1 & Risk of IHD & ICD-9, ICD-10 & $\begin{array}{l}\text { Yes for IHD }(H R=1.18 \text {, } \\
95 \% \mathrm{Cl}=1.06-1.31)\end{array}$ \\
\hline $\begin{array}{l}\text { Abouegylah } \\
(2019)^{51}\end{array}$ & 202 & Unknown & $\begin{array}{l}2000- \\
2014\end{array}$ & $\begin{array}{l}\text { Hospital-based } \\
\text { cohort }\end{array}$ & $\begin{array}{l}6.75 \text { for } \\
\text { left-sided } \\
\text { and } 7 \text { for } \\
\text { right-sided }\end{array}$ & $\begin{array}{l}\text { Cardiac outcomes } \\
\text { (cardiac ischaemia, } \\
\text { arrhythmia, heart } \\
\text { failure) }\end{array}$ & Did not specify & $\begin{array}{l}\text { Yes for ischaemia } \\
(\mathrm{OR}=0.08, p=0.021) \\
\text { and arrhythmias } \\
(\mathrm{OR}=0.05, p=0.005)\end{array}$ \\
\hline $\begin{array}{l}\text { Chang } \\
(2019)^{52}\end{array}$ & 1015 & Korea & $\begin{array}{l}2002- \\
2013\end{array}$ & $\begin{array}{l}\text { Population- } \\
\text { based cohort }\end{array}$ & 6.1 & $\begin{array}{l}\text { Acute coronary } \\
\text { events (newly } \\
\text { diagnosed IHD } \\
\text { that requires } \\
\text { anticoagulant } \\
\text { therapy or coronary } \\
\text { revascularisation, as } \\
\text { well as cardiac- } \\
\text { related death) }\end{array}$ & $\begin{array}{l}\text { ICD and } \\
\text { the medical } \\
\text { procedure } \\
\text { codes from the } \\
\text { Electronic Data } \\
\text { Interchange }\end{array}$ & $\begin{array}{l}\text { No }(H R=1.28 \\
95 \% C l=0.71-2.29 \\
p=0.413)\end{array}$ \\
\hline $\operatorname{Kim}(2019)^{55}$ & 660 & Korea & $\begin{array}{l}2005- \\
2015\end{array}$ & $\begin{array}{l}\text { Multicentre } \\
\text { cohort }\end{array}$ & 3.9 & $\begin{array}{l}\text { Cardiovascular } \\
\text { events (including } \\
\text { CVD mortality, MI, } \\
\text { heart failure, and } \\
\text { stroke) }\end{array}$ & Chart review & $\begin{array}{l}\text { No }(\mathrm{HR}=2.38, \\
95 \% \mathrm{Cl}=0.80-7.11 ; \\
\mathrm{p}=0.12) \\
\text { Yes for left-sided } \mathrm{RT}+ \\
\text { doxorubicin-equivalent } \\
\text { dose } \geq 250 \mathrm{mg} / \mathrm{m}^{2} \\
\text { vs. right-sided } \mathrm{RT}+ \\
\text { cumulative doxorubicin- } \\
\text { equivalent dose } \\
<250 \mathrm{mg} / \mathrm{m}^{2}(\mathrm{HR}=5.22, \\
95 \% \mathrm{Cl}=1.67-21.15 ; \\
\mathrm{p}=0.006)\end{array}$ \\
\hline $\begin{array}{l}\text { Boekel } \\
(2018)^{47}\end{array}$ & 14,645 & Netherlands & $\begin{array}{l}1970- \\
2009\end{array}$ & $\begin{array}{l}\text { Hospital-based } \\
\text { cohort }\end{array}$ & 14 & $\begin{array}{l}\text { Cardiovascular event } \\
\text { (CVD diagnosis or } \\
\text { death due to CVD) }\end{array}$ & ICD-10 & $\begin{array}{l}\text { Yes for left chest wall RT } \\
\text { vs. right breast RT for } \\
\mathrm{CVD}(\mathrm{HR}=1.83 \text {, } \\
95 \% \mathrm{Cl}=1.39-2.40) \text { and } \\
\mathrm{IHD}(\mathrm{HR}=2.57, \\
95 \% \mathrm{Cl}=1.61-4.11) \\
\text { No for left breast } \mathrm{RT} \text { vs. } \\
\text { right breast } \mathrm{RT} \text { for IHD } \\
(\mathrm{HR}=1.38,95 \% \mathrm{Cl}= \\
0.96-1.99) \\
\text { No for breast } \mathrm{RT} \text { without } \\
\mathrm{IMC}(\mathrm{HR}=1.11 \text {, } \\
95 \% \mathrm{Cl}=0.93-1.32)\end{array}$ \\
\hline $\begin{array}{l}\text { James } \\
(2018)^{53}\end{array}$ & 501 & $\begin{array}{l}\text { New } \\
\text { Zealand }\end{array}$ & $\begin{array}{l}2002- \\
2006\end{array}$ & $\begin{array}{l}\text { Hospital-based } \\
\text { cohort }\end{array}$ & 10.33 & $\begin{array}{l}\text { Cardiac toxicity } \\
\text { (Ml, admission for } \\
\text { chest pain, coronary } \\
\text { angiogram positivity, } \\
\text { and ischaemic } \\
\text { cardiac death) }\end{array}$ & ICD-10 & $\begin{array}{l}\text { No }(O R=1.565 \\
95 \% C l=0.7-3.6 \\
p=0.2885)\end{array}$ \\
\hline $\begin{array}{l}\text { Wadsten } \\
(2018)^{44}\end{array}$ & 2441 & Sweden & $\begin{array}{l}1992- \\
2012\end{array}$ & $\begin{array}{l}\text { Population- } \\
\text { based cohort }\end{array}$ & 8.8 & $\| H D$ & ICD-9 & $\begin{array}{l}\text { No }(\mathrm{HR}=0.85 \\
95 \% \mathrm{Cl}=0.53-1.37)\end{array}$ \\
\hline $\begin{array}{l}\text { Chang } \\
(2017)^{33}\end{array}$ & $\begin{array}{l}2577 \text { for } 1 \\
\text { institution which } \\
\text { was used for } \\
\text { acute coronary } \\
\text { event (YCCR) } \\
\text { and } 24,235 \\
\text { for nationwide } \\
\text { registry analysis } \\
\text { used for cardiac } \\
\text { mortality (KBCR) }\end{array}$ & Korea & $\begin{array}{l}1990- \\
2012\end{array}$ & $\begin{array}{l}\text { Population- } \\
\text { based cohort }\end{array}$ & $\begin{array}{l}7 \text { for YCCR } \\
\text { and } 7.9 \text { for } \\
\text { KBCR }\end{array}$ & $\begin{array}{l}\text { Acute coronary } \\
\text { event (death } \\
\text { resulting from heart } \\
\text { disease, or newly } \\
\text { diagnosed IHD) }\end{array}$ & ICD-10 & $\begin{array}{l}\text { No }(H R=1.16 \\
95 \% \mathrm{Cl}=0.59-2.29)\end{array}$ \\
\hline
\end{tabular}

Abbreviations: 95\% Cl = 95\% confidence interval; $\mathrm{AMl}=$ acute myocardial infarction; $\mathrm{CAD}=$ coronary artery disease; CHF = congestive heart failure; CVD = cardiovascular diseases; DCIS = ductal carcinoma in situ; EMERSE = Electronic Medical Record Search Engine; HR = hazard ratio; ICD = International Classification of Diseases; ICPC = International Classification of Primary Care; IHD = ischaemic heart disease; IMC = internal mammary chain; $\mathrm{IR}$ = incidence ratio; $\mathrm{KBCR}=$ Korean Breast Cancer Society; $\mathrm{Ml}=$ myocardial infarction; $\mathrm{MONICA}=$ monitoring or trends and determinants in cardiovascular disease; $\mathrm{OR}$ = odds ratio; $\mathrm{PCl}=$ percutaneous coronary intervention; $\mathrm{RCT}=$ randomised controlled trial; $\mathrm{RT}$ = radiotherapy; $\mathrm{YCCR}=$ Yonsei Cancer Center. 
Table 2. (cont'd)

\begin{tabular}{|c|c|c|c|c|c|c|c|c|}
\hline $\begin{array}{l}\text { Study (first } \\
\text { author) }\end{array}$ & Study population & Country & $\begin{array}{l}\text { Treatment } \\
\text { period }\end{array}$ & Study design & $\begin{array}{l}\text { Median or } \\
\text { mean follow- } \\
\text { up time, y }\end{array}$ & Endpoint(s) & Endpoint criteria & $\begin{array}{l}\text { Significant increased risk } \\
\text { of cardiovascular death }\end{array}$ \\
\hline $\begin{array}{l}\text { Dess } \\
(2017)^{43}\end{array}$ & 2126 & $\begin{array}{l}\text { United } \\
\text { States }\end{array}$ & $\begin{array}{l}1984- \\
2007\end{array}$ & $\begin{array}{l}\text { Single-centre } \\
\text { cohort }\end{array}$ & 9.3 & $\begin{array}{l}\text { 10-year rate of } \\
\text { ischaemic cardiac } \\
\text { events (AMI, } \\
\text { coronary artery } \\
\text { bypass grafting, } \\
\text { angioplasty or stent } \\
\text { placement, diagnosis } \\
\text { of CAD) }\end{array}$ & ICD, EMERSE & $\begin{array}{l}\text { No }(H R=1.21,95 \% \mathrm{Cl}= \\
0.79-1.85, p=0.37)\end{array}$ \\
\hline $\begin{array}{l}\text { Rehammar } \\
(2017)^{46}\end{array}$ & 19,464 & Denmark & $\begin{array}{l}1977- \\
2005\end{array}$ & $\begin{array}{l}\text { Population- } \\
\text { based cohort }\end{array}$ & 10.5 & Cardiac disease & ICD-10 & $\begin{array}{l}\text { Yes }(H R=1.11 \\
95 \% \mathrm{Cl}=1.03-1.20 \\
p=0.005)\end{array}$ \\
\hline $\begin{array}{l}\text { Wollschläger } \\
(2017)^{57}\end{array}$ & 4474 & Germany & $\begin{array}{l}1998- \\
2008\end{array}$ & $\begin{array}{l}\text { Multicentre } \\
\text { cohort study }\end{array}$ & 8.3 & $\begin{array}{l}\text { Cardiac event } \\
\text { (Ml, angina, CHF, } \\
\text { arrhythmia, or } \\
\text { valvular heart } \\
\text { disease) }\end{array}$ & ICD-10 & $\begin{array}{l}\text { No }(\mathrm{HR}=1.07 \\
95 \% \mathrm{Cl}=0.89-1.29)\end{array}$ \\
\hline $\begin{array}{l}\text { Boerman } \\
(2014)^{45}\end{array}$ & 334 & Netherlands & $\begin{array}{l}1970- \\
2007\end{array}$ & $\begin{array}{l}\text { Matched case- } \\
\text { control study }\end{array}$ & 9 & $\begin{array}{l}\text { CHF (acute and } \\
\text { chronic), vascular } \\
\text { cardiac diseases } \\
\text { (unstable and stable } \\
\text { angina pectoris, } \\
\text { AMl, other chronic } \\
\text { IHDs [coronary artery } \\
\text { sclerosis], transient } \\
\text { ischaemic attack, } \\
\text { and cerebrovascular } \\
\text { attack), and other } \\
\text { cardiac diseases } \\
\text { (atrial fibrillation, } \\
\text { paroxysmal } \\
\text { tachycardia, } \\
\text { nonrheumatic valve } \\
\text { disease) }\end{array}$ & ICPC version 1 & $\begin{array}{l}\text { No for } \mathrm{CHF}(\mathrm{HR}=0.98 \text {, } \\
95 \% \mathrm{Cl}=0.3-3.6) \text {, } \\
\text { vascular cardiac diseases } \\
\text { (HR=0.7, } 95 \% \mathrm{Cl}= \\
\text { 0.3-1.4), or other cardiac } \\
\text { diseases }(\mathrm{HR}=0.8 \text {, } \\
95 \% \mathrm{Cl}=0.4-1.7)\end{array}$ \\
\hline $\begin{array}{l}\text { Boekel } \\
(2014)^{7}\end{array}$ & $\begin{array}{l}2899 \text { (DCIS } \\
\text { patients only) }\end{array}$ & Netherlands & $\begin{array}{l}1989- \\
2004\end{array}$ & $\begin{array}{l}\text { Population- } \\
\text { based cohort }\end{array}$ & 10 & $\begin{array}{l}\text { Ml, other IHDs, } \\
\text { other heart disease, } \\
\text { pericarditis, valvular } \\
\text { dysfunction, } \\
\text { cardiomyopathy, } \\
\text { arrhythmia, } \\
\text { CHF, and/or } \\
\text { cerebrovascular } \\
\text { disease }\end{array}$ & ICD-10 & $\begin{array}{l}\text { No }(\mathrm{HR}=0.94 \\
95 \% \mathrm{Cl}=0.67-1.32)\end{array}$ \\
\hline $\begin{array}{l}\text { Soran } \\
(2014)^{50}\end{array}$ & 602 & $\begin{array}{l}\text { United } \\
\text { States }\end{array}$ & $\begin{array}{l}1986- \\
2007\end{array}$ & $\begin{array}{l}\text { Hospital-based } \\
\text { cohort }\end{array}$ & 7.5 & $\begin{array}{l}\text { Major cardiac events } \\
\text { (chest pain, Ml, } \\
\text { atherosclerosis, } \\
\text { angina, stroke) }\end{array}$ & $\begin{array}{l}\text { Self-reported } \\
\text { questionnaire }\end{array}$ & $\begin{array}{l}\text { No for chest pain } \\
(p=0.9), M I(p=0.1), \\
\text { atherosclerosis }(p=0.07) \text {, } \\
\text { angina }(p=0.3) \text {, or stroke } \\
(p=0.1)\end{array}$ \\
\hline $\begin{array}{l}\text { Darby } \\
(2013)^{40}\end{array}$ & $\begin{array}{l}2168 \text { (963 } \\
\text { cases and } 1205 \\
\text { controls) }\end{array}$ & $\begin{array}{l}\text { United } \\
\text { States }\end{array}$ & $\begin{array}{l}1958- \\
2001 \\
\text { (Sweden), } \\
1977- \\
2000 \\
\text { (Denmark) }\end{array}$ & $\begin{array}{l}\text { Population- } \\
\text { based } \\
\text { case-control }\end{array}$ & $\begin{array}{l}\text { Did not } \\
\text { specify }\end{array}$ & $\begin{array}{l}\text { Major coronary } \\
\text { events (Ml, coronary } \\
\text { revascularisation, or } \\
\text { death from IHD) }\end{array}$ & ICD-10 & Yes $(p=0.002)$ \\
\hline $\begin{array}{l}\text { Bouillon } \\
(2011)^{10}\end{array}$ & 3038 & France & $\begin{array}{l}1954- \\
1984\end{array}$ & $\begin{array}{l}\text { Single-centre } \\
\text { cohort }\end{array}$ & 28 & $\begin{array}{l}\text { Cardiac diseases } \\
\text { (pericarditis, } \\
\text { myocarditis, valvular } \\
\text { heart diseases, IHD, } \\
\text { heart failure) }\end{array}$ & $\begin{array}{l}\text { ICD-8, ICD-9, } \\
\text { ICD-10 }\end{array}$ & $\begin{array}{l}\text { No (1.28-fold more likely; } \\
95 \% \mathrm{Cl}=0.92-1.78)\end{array}$ \\
\hline $\begin{array}{l}\text { Haque } \\
(2011)^{49}\end{array}$ & 340 & $\begin{array}{l}\text { United } \\
\text { States }\end{array}$ & $\begin{array}{l}1990- \\
1994\end{array}$ & $\begin{array}{l}\text { Population- } \\
\text { based cohort }\end{array}$ & 10 & $\begin{array}{l}\text { CVD events that } \\
\text { were serious } \\
\text { enough to require } \\
\text { hospitalisation (IHD, } \\
\text { AMl, angina, and } \\
\text { CVD) }\end{array}$ & ICD-9, ICD-10 & $\begin{array}{l}\text { Yes }(\mathrm{HR}=1.53 \\
95 \% \mathrm{Cl}=1.06-2.21)\end{array}$ \\
\hline Park $(2011)^{12}$ & $\begin{array}{l}129 \text { (DCIS } \\
\text { patients only) }\end{array}$ & $\begin{array}{l}\text { United } \\
\text { States }\end{array}$ & $\begin{array}{l}1986- \\
2002\end{array}$ & $\begin{array}{l}\text { Single-centre } \\
\text { cohort }\end{array}$ & 8.2 & $\begin{array}{l}\text { CAD, MI, CHF, } \\
\text { chronic IHD, } \\
\text { arrhythmia, valvular } \\
\text { disease and/or } \\
\text { cardiomyopathy }\end{array}$ & Did not specify & $\begin{array}{l}\text { No, } 13.5 \% \text { of left-sided } \\
\text { RT patients developed } \\
\text { a cardiovascular event } \\
\text { compared with } 7 \% \text { of } \\
\text { right-sided patients } \\
(\mathrm{p}=0.25)\end{array}$ \\
\hline
\end{tabular}


Table 2. (cont'd)

\begin{tabular}{|c|c|c|c|c|c|c|c|c|}
\hline $\begin{array}{l}\text { Study (first } \\
\text { author) }\end{array}$ & Study population & Country & $\begin{array}{l}\text { Treatment } \\
\text { period }\end{array}$ & Study design & $\begin{array}{l}\text { Median or } \\
\text { mean follow- } \\
\text { up time, y }\end{array}$ & Endpoint(s) & Endpoint criteria & $\begin{array}{l}\text { Significant increased risk } \\
\text { of cardiovascular death }\end{array}$ \\
\hline $\begin{array}{l}\text { McGale } \\
(2011)^{11}\end{array}$ & 34,825 & $\begin{array}{l}\text { Sweden } \\
\text { and } \\
\text { Denmark }\end{array}$ & $\begin{array}{l}1976- \\
2006\end{array}$ & $\begin{array}{l}\text { Population- } \\
\text { based cohort }\end{array}$ & $\begin{array}{l}\text { Not } \\
\text { specified. }\end{array}$ & Heart disease & $\begin{array}{l}\text { ICD-8, ICD-9, } \\
\text { ICD-10 }\end{array}$ & $\begin{array}{l}\text { Yes }(\mathrm{IR}=1.08 \\
95 \% \mathrm{Cl}=1.02-1.15 \\
\mathrm{p}=0.01)\end{array}$ \\
\hline Gutt $(2008)^{16}$ & 41 & $\begin{array}{l}\text { United } \\
\text { States }\end{array}$ & $\begin{array}{l}1980- \\
1994\end{array}$ & $\begin{array}{l}\text { Single-centre } \\
\text { cohort }\end{array}$ & $\begin{array}{l}7.9 \text { for } \\
\text { left-sided } \\
\text { and } 11.3 \text { for } \\
\text { right-sided }\end{array}$ & $\begin{array}{l}\text { MI, CHF, CAD, } \\
\text { arrhythmia, chest } \\
\text { pain, and/or valvular } \\
\text { disease }\end{array}$ & Not specified & $\begin{array}{l}\text { No for } C A D(p=0.6), M l \\
(p=0.96), C H F \\
(p=0.73) \text {, arrhythmia } \\
(p=0.31) \text {, or valvular } \\
\text { disease }(p=0.89)\end{array}$ \\
\hline $\begin{array}{l}\text { Borger } \\
(2007)^{18}\end{array}$ & 1601 & Netherlands & $\begin{array}{l}1980- \\
1993\end{array}$ & $\begin{array}{l}\text { Multicentre } \\
\text { cohort }\end{array}$ & 16 & $\begin{array}{l}\text { CVD (including IHD } \\
\text { and other heart } \\
\text { diseases) }\end{array}$ & ICD-9 & $\begin{array}{l}\text { Yes for CVD }(H R=1.38 \text {, } \\
95 \% \mathrm{Cl}=1.05-1.81) \text { and } \\
\text { non- } \mathrm{HD}(\mathrm{HR}=1.53 \text {, } \\
95 \% \mathrm{Cl}=1.09-2.15) . \\
\text { No for } \mathrm{IHD}(\mathrm{HR}=1.35 \text {, } \\
95 \% \mathrm{Cl}=0.93-1.98)\end{array}$ \\
\hline $\begin{array}{l}\text { Doyle } \\
(2007)^{48}\end{array}$ & 25,653 & $\begin{array}{l}\text { United } \\
\text { States }\end{array}$ & $\begin{array}{l}1992- \\
2000\end{array}$ & $\begin{array}{l}\text { Population- } \\
\text { based cohort }\end{array}$ & 13 as max & $\begin{array}{l}\text { MI, ischaemia, CHF, } \\
\text { and other heart } \\
\text { disease (myocarditis, } \\
\text { arrhythmia, and } \\
\text { valvular disease) }\end{array}$ & ICD-9 & $\begin{array}{l}\text { No for } \mathrm{Ml} / \text { ischaemia } \\
(\mathrm{HR}=1.03 \text {, } \\
95 \% \mathrm{Cl}=0.93-1.13) \text { or for } \\
\mathrm{Ml} \text { alone }(\mathrm{HR}=0.99 \\
95 \% \mathrm{Cl}=0.87-1.11)\end{array}$ \\
\hline $\begin{array}{l}\text { Jagsi } \\
(2007)^{41}\end{array}$ & 828 & $\begin{array}{l}\text { United } \\
\text { States }\end{array}$ & $\begin{array}{l}1984- \\
2000\end{array}$ & $\begin{array}{l}\text { Single-centre } \\
\text { cohort }\end{array}$ & 6.8 & $\begin{array}{l}\text { Cardiac events } \\
\text { (CAD or Ml requiring } \\
\text { intervention) }\end{array}$ & ICD-9 & $\begin{array}{l}\text { Yes for } \mathrm{Ml}(\mathrm{HR}=7.92 \\
95 \% \mathrm{Cl}=1.01-62.28 \\
\mathrm{p}=0.05)\end{array}$ \\
\hline $\begin{array}{l}\text { Paszat } \\
(2007)^{20}\end{array}$ & 619 & Canada & $\begin{array}{l}1982- \\
1988\end{array}$ & $\begin{array}{l}\text { Population- } \\
\text { based cohort }\end{array}$ & $\begin{array}{l}13.5 \\
\text { minimum }\end{array}$ & $\begin{array}{l}\text { AMl and death due } \\
\text { to AMl }\end{array}$ & Chart review & $\begin{array}{l}\text { No }(\mathrm{HR}=1.42, \\
95 \% \mathrm{Cl}=0.92-2.17)\end{array}$ \\
\hline $\begin{array}{l}\text { Harris } \\
(2006)^{22}\end{array}$ & 961 & $\begin{array}{l}\text { United } \\
\text { States }\end{array}$ & $\begin{array}{l}1977- \\
1994\end{array}$ & $\begin{array}{l}\text { Single-centre } \\
\text { cohort }\end{array}$ & 12 & $\begin{array}{l}\text { Chest pain, CAD, } \\
\text { MI, CHF and chronic } \\
\Vdash H D \text {, arrhythmia and } \\
\text { palpations and/or } \\
\text { valvular disorders }\end{array}$ & Not specified & $\begin{array}{l}\text { Yes for chest pain } \\
(\mathrm{IR}=2.1,95 \% \mathrm{Cl}=1.5-2.9 ; \\
\mathrm{p}<0.001), \mathrm{CAD}(\mathrm{IR}=2.7 \\
95 \% \mathrm{Cl}=1.7-4.5 ; \\
\mathrm{p}<0.001) \text { and } \mathrm{Ml} \\
(\mathrm{I}=3.1,95 \% \mathrm{Cl}=1.5-6.5 ; \\
\mathrm{p}=0.002) \\
\text { No for } \mathrm{CHF} / \text { chronic IHD } \\
(\mathrm{I}=1.2,95 \% \mathrm{Cl}=0.8-2.0 ; \\
p=0.37), \text { arrhythmia } \\
(\mathrm{I}=1.3,95 \% \mathrm{Cl}=0.8-1.9 ; \\
\mathrm{p}=0.21) \text { and valvular } \\
\text { disorders }(\mathrm{IR}=0.85 \\
95 \% \mathrm{Cl}=0.6-1.3 ; \\
\mathrm{p}=0.70)\end{array}$ \\
\hline Patt $(2005)^{42}$ & 16,270 & $\begin{array}{l}\text { United } \\
\text { States }\end{array}$ & $\begin{array}{l}1986- \\
1993\end{array}$ & $\begin{array}{l}\text { Population- } \\
\text { based cohort }\end{array}$ & 9.5 & $\begin{array}{l}\text { IHD, valvular heart } \\
\text { disease, CHF, and/ } \\
\text { or conduction } \\
\text { abnormalities }\end{array}$ & ICD-9 & $\begin{array}{l}\text { No for } \mathrm{HD}(\mathrm{HR}=1.05 \text {, } \\
95 \% \mathrm{Cl}=0.94-1.16) \text {, } \\
\text { valvular disease } \\
(\mathrm{HR}=1.07,95 \% \mathrm{Cl}= \\
0.89-1.30), \mathrm{CHF} \\
(\mathrm{HR}=1.05,95 \% \mathrm{Cl}= \\
0.95-1.17), \text { and } \\
\text { conduction abnormalities } \\
(\mathrm{HR}=1.07,95 \% \mathrm{Cl}= \\
0.96-1.19)\end{array}$ \\
\hline $\begin{array}{l}\text { Vallis } \\
(2002)^{24}\end{array}$ & 2128 & Canada & $\begin{array}{l}1982- \\
1988\end{array}$ & $\begin{array}{l}\text { Single-centre } \\
\text { cohort }\end{array}$ & 10.2 & $\begin{array}{l}\text { Ml and/or other } \\
\text { cardiac events }\end{array}$ & $\begin{array}{l}\text { ICD-9 combined } \\
\text { with the } \\
\text { multinational } \\
\text { MONICA criteria }\end{array}$ & $\begin{array}{l}\text { No for } \mathrm{Ml}(\mathrm{p}=0.66) \text { or } \\
\text { other cardiac events } \\
(\mathrm{p}=0.24)\end{array}$ \\
\hline
\end{tabular}


Adjuvant Breast Cancer RT and Cardiac Events

Table 3. Studies assessing the risk of cardiovascular mortality in patients who received RT compared with those who did not receive RT.

\begin{tabular}{|c|c|c|c|c|c|c|c|c|}
\hline $\begin{array}{l}\text { Study (first } \\
\text { author) }\end{array}$ & $\begin{array}{l}\text { Study } \\
\text { population }\end{array}$ & Country & $\begin{array}{l}\text { Treat- } \\
\text { ment } \\
\text { period }\end{array}$ & Study design & $\begin{array}{l}\text { Median or } \\
\text { mean follow- } \\
\text { up time, y }\end{array}$ & Endpoint(s) & Endpoint criteria & $\begin{array}{l}\text { Significant increased risk of } \\
\text { cardiovascular death }\end{array}$ \\
\hline $\begin{array}{l}\text { Boekel } \\
(2020)^{58}\end{array}$ & 408 & Netherlands & $\begin{array}{l}1976- \\
2009\end{array}$ & $\begin{array}{l}\text { Population- } \\
\text { based } \\
\text { case-control }\end{array}$ & $\begin{array}{l}\text { Did not } \\
\text { specify }\end{array}$ & Heart failure & Chart review & $\begin{array}{l}\text { No for RT vs. MHD between } \\
0 \text { and } 1 \mathrm{~Gy}(\mathrm{RR}=1.4 \text {, } \\
95 \% \mathrm{Cl}=0.73-2.8 ; \mathrm{p}=0.51)\end{array}$ \\
\hline Chou $(2020)^{59}$ & 27,466 & Taiwan & $\begin{array}{l}2007- \\
2014\end{array}$ & $\begin{array}{l}\text { Population- } \\
\text { based cohort }\end{array}$ & $\begin{array}{l}\text { Did not } \\
\text { specify }\end{array}$ & $\begin{array}{l}\text { MACE, identified } \\
\text { by emergency } \\
\text { visit claims or } \\
\text { inpatient data of } \\
\text { IHD, CHF, acute } \\
\text { ischaemic stroke, } \\
\text { and intracranial } \\
\text { haemorrhage }\end{array}$ & $\begin{array}{l}\text { ICD-9 Clinical } \\
\text { Modification }\end{array}$ & $\begin{array}{l}\text { No, } R T+\text { hormone group } \\
\text { had a significantly lower risk } \\
\text { than the hormone only group } \\
(\mathrm{HR}=0.609,95 \% \mathrm{Cl}= \\
0.430-0.862 ; \mathrm{p}=0.005)\end{array}$ \\
\hline $\begin{array}{l}\text { Henson } \\
(2020)^{5}\end{array}$ & $1,934,248$ & Multinational & $\begin{array}{l}1987- \\
2002\end{array}$ & $\begin{array}{l}\text { Population- } \\
\text { based cohort }\end{array}$ & 6.7 & Cardiac mortality & Did not specify & $\begin{array}{l}\text { No, there was a decreased } \\
\text { risk }(\mathrm{RR}=0.94,95 \% \mathrm{Cl}= \\
0.92-0.95) \\
\text { Yes for } \mathrm{RT} \text { after mastectomy } \\
(\mathrm{RR}=1.24,95 \% \mathrm{Cl}= \\
1.19-1.30)\end{array}$ \\
\hline $\begin{array}{l}\text { Killander } \\
(2020)^{54}\end{array}$ & 1144 & Sweden & $\begin{array}{l}1991- \\
1997\end{array}$ & $\mathrm{RCT}$ & 21.3 & $\begin{array}{l}\text { Cardiac morbidity and } \\
\text { mortality }\end{array}$ & $\begin{array}{l}\text { ICD-8, ICD-9, } \\
\text { ICD-10 }\end{array}$ & $\begin{array}{l}\text { No, } 12.4 \% \text { incidence of } \\
\text { cardiac mortality in no RT } \\
\text { group vs. } 13 \% \text { in RT group } \\
(p=0.8)\end{array}$ \\
\hline Lee $(2020)^{60}$ & 91,227 & Korea & $\begin{array}{l}2007- \\
2013\end{array}$ & $\begin{array}{l}\text { Population- } \\
\text { based cohort }\end{array}$ & 3.1 & $\mathrm{CHF}$ & ICD-10 & $\begin{array}{l}\text { No, risk was lower when } \\
\text { analysed from } \mathrm{BC} \text { diagnosis } \\
\text { (HR=0.734, 95\% } \mathrm{Cl}= \\
0.652-0.826) \text { and when } \\
\text { analysed from } 2 \text { years after } \\
\mathrm{BC} \text { diagnosis }(\mathrm{HR}=0.688 \text {, } \\
95 \% \mathrm{Cl}=0.583-0.811)\end{array}$ \\
\hline $\begin{array}{l}\text { Lawrenson } \\
(2019)^{61}\end{array}$ & 3528 & $\begin{array}{l}\text { New } \\
\text { Zealand }\end{array}$ & $\begin{array}{l}1995- \\
2013\end{array}$ & $\begin{array}{l}\text { Population- } \\
\text { based cohort }\end{array}$ & $\begin{array}{l}\text { 10-year } \\
\text { follow-up } \\
\text { period }\end{array}$ & $\begin{array}{l}\text { Cardiovascular events } \\
\text { and cardiovascular } \\
\text { mortality }\end{array}$ & ICD-9 & $\begin{array}{l}\text { No }(\mathrm{HR}=0.75,95 \% \mathrm{Cl}= \\
0.47-1.19)\end{array}$ \\
\hline Obi $(2018)^{27}$ & 2951 & Germany & $\begin{array}{l}2001- \\
2005\end{array}$ & $\begin{array}{l}\text { Population- } \\
\text { based } \\
\text { case-control }\end{array}$ & 11.9 & Cardiac mortality & ICD-10 & $\begin{array}{l}\text { No }(\mathrm{HR}=1.57,95 \% \mathrm{Cl}= \\
0.75-3.29)\end{array}$ \\
\hline Leung $(2016)^{62}$ & 5132 & Taiwan & $\begin{array}{l}2000- \\
2010\end{array}$ & $\begin{array}{l}\text { Population- } \\
\text { based } \\
\text { case-control } \\
\text { study }\end{array}$ & 3.5 & $\begin{array}{l}\text { Death due to IHD, } \\
\text { valvular heart } \\
\text { disease, CHF, and/ } \\
\text { or conduction } \\
\text { abnormalities }\end{array}$ & ICD-9 & $\begin{array}{l}\text { No }(\mathrm{HR}=1.40,95 \% \mathrm{Cl}= \\
0.80-2.47, \mathrm{p}=0.13) \\
\text { No for } \mathrm{IHD}(\mathrm{HR}=1.41 \text {, } \\
95 \% \mathrm{Cl}=0.56-3.56) \text {, valvular } \\
\text { heart disease }(\mathrm{HR}=0.57, \\
95 \% \mathrm{Cl}=0.08-4.26), \mathrm{CHF} \\
(\mathrm{HR}=1.56,95 \% \mathrm{Cl}= \\
\text { 0.66-3.69), and conduction } \\
\text { abnormalities (HR=2.77, } \\
95 \% \mathrm{Cl}=0.95-8.06)\end{array}$ \\
\hline Ye $(2015)^{38}$ & 6515 & $\begin{array}{l}\text { United } \\
\text { States }\end{array}$ & $\begin{array}{l}1990- \\
1997\end{array}$ & $\begin{array}{l}\text { Population- } \\
\text { based cohort }\end{array}$ & 15 & Cardiac mortality & $\begin{array}{l}\text { Cause of death } \\
\text { codes }\end{array}$ & $\begin{array}{l}\text { No }(H R=0.57,95 \% C l= \\
0.47-0.69 ; p<0.0001)\end{array}$ \\
\hline Boekel $(2014)^{7}$ & $\begin{array}{l}10,365 \text { (DCIS } \\
\text { patients only) }\end{array}$ & Netherlands & $\begin{array}{l}1989- \\
2004\end{array}$ & $\begin{array}{l}\text { Population- } \\
\text { based cohort }\end{array}$ & 10 & $\begin{array}{l}\text { Death due to MI, } \\
\text { other IHDs, other } \\
\text { heart disease, } \\
\text { pericarditis, valvular } \\
\text { dysfunction, } \\
\text { cardiomyopathy, } \\
\text { arrhythmia, CHF, and/ } \\
\text { or cerebrovascular } \\
\text { disease }\end{array}$ & ICD-10 & $\begin{array}{l}\text { No significant difference } \\
\text { in cardiovascular death } \\
\text { between patients who } \\
\text { received left-sided (HR=0.89, } \\
95 \% \mathrm{Cl}=0.48-1.66) \text { or right- } \\
\text { sided } \mathrm{RT}(\mathrm{HR}=1.29 \text {, } \\
95 \% \mathrm{Cl}=0.72-2.31) \text { vs. } \\
\text { surgery only }\end{array}$ \\
\hline $\begin{array}{l}\text { Killander } \\
(2014)^{63}\end{array}$ & 1110 & Sweden & $\begin{array}{l}1978- \\
1985\end{array}$ & Two RCTs & Not specified & $\begin{array}{l}\text { 25-year mortality } \\
\text { rates due to IHD, } \\
\text { CHF, dysrhythmia, } \\
\text { and non-rheumatic } \\
\text { valvular and/or } \\
\text { pericardial disease }\end{array}$ & $\begin{array}{l}\text { ICD-8, ICD-9, } \\
\text { ICD-10 }\end{array}$ & $\begin{array}{l}\text { Yes, when RT was combined } \\
\text { with cyclophosphamide } \\
\text { in premenopausal women } \\
(p=0.04) \text { or tamoxifen in } \\
\text { postmenopausal women } \\
(p=0.005)\end{array}$ \\
\hline
\end{tabular}

Abbreviations: 95\% Cl = 95\% confidence interval; $\mathrm{AMI}=$ acute myocardial infarction; $\mathrm{BC}=$ breast cancer; $\mathrm{BCS}=$ breast-conserving surgery; Chemo = chemotherapy; $\mathrm{CHF}=$ congestive heart failure; $\mathrm{CVD}=$ cardiovascular diseases; $\mathrm{DCIS}=$ ductal carcinoma in situ; $\mathrm{HR}=$ hazard ratio; $I C D=$ International Classification of Diseases; $I H D=$ ischaemic heart disease; MACE = major adverse cardiac events; $\mathrm{MHD}=$ mean heart dose; $\mathrm{Ml}=$ myocardial infarction; $\mathrm{RCT}=$ randomised controlled trial; $\mathrm{RR}=$ risk ratio; $\mathrm{RT}$ = radiotherapy; $\mathrm{SMR}=$ standardised mortality ratio. 
Table 3. (cont'd)

\begin{tabular}{|c|c|c|c|c|c|c|c|c|}
\hline $\begin{array}{l}\text { Study (first } \\
\text { author) }\end{array}$ & $\begin{array}{l}\text { Study } \\
\text { population }\end{array}$ & Country & $\begin{array}{l}\text { Treat- } \\
\text { ment } \\
\text { period }\end{array}$ & Study design & $\begin{array}{l}\text { Median or } \\
\text { mean follow- } \\
\text { up time, y }\end{array}$ & Endpoint(s) & Endpoint criteria & $\begin{array}{l}\text { Significant increased risk of } \\
\text { cardiovascular death }\end{array}$ \\
\hline $\begin{array}{l}\text { Bouillon } \\
(2011)^{10}\end{array}$ & 4456 & France & $\begin{array}{l}1954- \\
1984\end{array}$ & $\begin{array}{l}\text { Single-centre } \\
\text { cohort }\end{array}$ & 28 & $\begin{array}{l}\text { Death due to vascular } \\
\text { diseases (pulmonary } \\
\text { heart diseases, } \\
\text { hypertensive } \\
\text { diseases, } \\
\text { cerebrovascular } \\
\text { diseases, diseases } \\
\text { of arteries, arterioles } \\
\text { and capillaries) and } \\
\text { cardiac diseases } \\
\text { (pericarditis, } \\
\text { myocarditis, valvular } \\
\text { heart diseases, IHD, } \\
\text { heart failure) }\end{array}$ & $\begin{array}{l}\text { ICD-8, ICD-9, } \\
\text { ICD-10 }\end{array}$ & $\begin{array}{l}\text { Yes for cardiac diseases } \\
\text { (1.76-fold more likely, } \\
95 \% \mathrm{Cl}=1.34-2.31) \\
\text { No for vascular diseases } \\
\text { (1.33-fold more likely, } \\
95 \% \mathrm{Cl}=0.99-1.80)\end{array}$ \\
\hline Wang $(2011)^{14}$ & 1242 & Australia & 1995 & $\begin{array}{l}\text { Population- } \\
\text { based cohort }\end{array}$ & Not specified & $\begin{array}{l}\text { 10-year cardiac } \\
\text { mortality }\end{array}$ & $\begin{array}{l}\text { Death certificates } \\
\text { codes }\end{array}$ & $\begin{array}{l}\text { No, higher cumulative } \\
\text { mortality due to cardiac } \\
\text { causes in non-RT group, } \\
\text { compared with left-sided and } \\
\text { right-sided RT groups } \\
(p=0.001)\end{array}$ \\
\hline $\begin{array}{l}\text { Roychoudhuri } \\
(2007)^{21}\end{array}$ & 20,871 & $\begin{array}{l}\text { South East } \\
\text { England }\end{array}$ & $\begin{array}{l}1971- \\
1988\end{array}$ & $\begin{array}{l}\text { Population- } \\
\text { based cohort }\end{array}$ & 18.5 & IHD and other CVDs & ICD-9, ICD-10 & $\begin{array}{l}\text { No for IHD mortality } \\
(\mathrm{HR}=1.28,95 \% \mathrm{Cl}= \\
0.96-1.70 ; \mathrm{p}=0.092) \text { and } \\
\mathrm{CVD} \text { mortality }(\mathrm{HR}=1.02 \text {, } \\
95 \% \mathrm{Cl}=0.85-1.23 ; \\
\mathrm{p}=0.806) \text { when comparing } \\
\text { right-sided irradiated patients } \\
\text { to right-sided non-irradiated } \\
\text { patients } \\
\text { Yes for } \mathrm{IHD} \text { mortality } \\
(\mathrm{HR}=1.59,95 \% \mathrm{Cl}= \\
1.21-2.08) \text { and } \mathrm{CVD} \text { mortality } \\
(\mathrm{HR}=1.27,95 \% \mathrm{Cl}= \\
1.07-1.51) \text { when comparing } \\
\text { left-sided } \mathrm{RT} \text { patients to right- } \\
\text { sided patients with no } \mathrm{RT}\end{array}$ \\
\hline $\begin{array}{l}\text { Clarke, et al } \\
(2005)^{64}\end{array}$ & 42,080 & Multinational & $\begin{array}{l}1995- \\
2000\end{array}$ & $\mathrm{RCT}$ & $\begin{array}{l}\text { 5-, 10-, and } \\
15 \text {-year } \\
\text { mortality } \\
\text { rates }\end{array}$ & Cardiac mortality & ICD-9 & Yes $(R R=1.27 ; 2 p=0.0001)$ \\
\hline Ragaz $(2005)^{65}$ & $\begin{array}{l}318 \text { (164 in } \\
\text { Chemo + RT, } \\
\text { and } 154 \text { in } \\
\text { Chemo only) }\end{array}$ & Canada & $\begin{array}{l}1979- \\
1986\end{array}$ & $\mathrm{RCT}$ & 20.75 & Cardiac death & Did not specify & $\begin{array}{l}\text { No, } 1.8 \% \text { death rate in } \\
\text { Chemo + RT and } 0.6 \% \text { in } \\
\text { Chemo alone }(p=0.622)\end{array}$ \\
\hline $\begin{array}{l}\text { Woodward } \\
(2003)^{66}\end{array}$ & 1493 & $\begin{array}{l}\text { United } \\
\text { States }\end{array}$ & $\begin{array}{l}1975- \\
1994\end{array}$ & $\begin{array}{l}5 \text { separate } \\
\text { clinical trials }\end{array}$ & 10 & $\begin{array}{l}\text { 10-year rate of death } \\
\text { from Ml }\end{array}$ & Did not specify & $\begin{array}{l}\text { No (10-year death rate from } \\
\text { Ml was } 2.4 \% \text { in RT patients } \\
\text { vs. } 0.5 \% \text { in no RT patients } \\
[p=0.057])\end{array}$ \\
\hline Hojris (1999) $)^{67}$ & 3046 & Denmark & $\begin{array}{l}1982- \\
1990\end{array}$ & Two RCTs & 9.75 & IHD (including AMI) & ICD-8, ICD-10 & $\begin{array}{l}\text { No }(H R=1.12,95 \% \mathrm{Cl}= \\
0.73-2.95)\end{array}$ \\
\hline \multirow[t]{2}{*}{$\begin{array}{l}\text { Gyenes } \\
(1998)^{68}\end{array}$} & 960 & Sweden & $\begin{array}{l}1971- \\
1976\end{array}$ & $\begin{array}{l}\text { Multicentre } \\
\text { case-control } \\
\text { study }\end{array}$ & 20 & $\begin{array}{l}\text { Death due to IHD } \\
\text { and/or CVD }\end{array}$ & ICD-9 & $\begin{array}{l}\text { Yes for IHD mortality } \\
(\mathrm{HR}=2.5,95 \% \mathrm{Cl}=1.1-5.7 ; \\
\mathrm{p}=0.03) \text { and } \mathrm{CVD} \text { mortality } \\
(\mathrm{HR}=2.0,95 \% \mathrm{Cl}=1.0-3.9 ; \\
\mathrm{p}=0.04) \text { in high dose-volume } \\
\text { RT patients compared with } \\
\text { surgical controls } \\
\text { No for IHD mortality and CVD } \\
\text { mortality in low dose-volume } \\
\text { RT patients compared with } \\
\text { surgical controls }\end{array}$ \\
\hline & & & & & & & & $\begin{array}{l}\text { Note: High dose-volume was } \\
\text { defined as using left-sided } \\
\text { tangential } 60 \text { Co fields. Low } \\
\text { dose-volume was defined as } \\
\text { using right-sided tangential } \\
60 \text { Co fields. Intermediate } \\
\text { dose was defined as using } \\
\text { electron techniques }\end{array}$ \\
\hline
\end{tabular}


Table 3. (cont'd)

\begin{tabular}{|c|c|c|c|c|c|c|c|c|}
\hline $\begin{array}{l}\text { Study (first } \\
\text { author) }\end{array}$ & $\begin{array}{l}\text { Study } \\
\text { population }\end{array}$ & Country & $\begin{array}{l}\text { Treat- } \\
\text { ment } \\
\text { period }\end{array}$ & Study design & $\begin{array}{l}\text { Median or } \\
\text { mean follow- } \\
\text { up time, y }\end{array}$ & Endpoint(s) & Endpoint criteria & $\begin{array}{l}\text { Significant increased risk of } \\
\text { cardiovascular death }\end{array}$ \\
\hline $\begin{array}{l}\text { Rutqvist } \\
(1998)^{69}\end{array}$ & 5680 & Sweden & $\begin{array}{l}1976- \\
1987\end{array}$ & $\begin{array}{l}\text { Hospital-based } \\
\text { case-cohort }\end{array}$ & 9 & AMl & Did not specify & $\begin{array}{l}\text { No for } \mathrm{RT}+\mathrm{BCS} \text { vs. } \\
\text { mastectomy alone }(\mathrm{HR}=0.4 \text {, } \\
95 \% \mathrm{Cl}=0.2-1.1)\end{array}$ \\
\hline $\begin{array}{l}\text { Cuzick } \\
(1994)^{29}\end{array}$ & 7941 & Multinational & $\begin{array}{l}1949- \\
1974\end{array}$ & Multiple RCTs & $>10$ & Cardiac mortality & ICD-9 & $\begin{array}{l}\text { Yes (SMR=1.62, 95\% } \\
\mathrm{Cl}=1.25-2.1 ; p<0.001)\end{array}$ \\
\hline $\begin{array}{l}\text { Houghton } \\
(1994)^{30}\end{array}$ & 2800 & $\begin{array}{l}\text { United } \\
\text { Kingdom }\end{array}$ & $\begin{array}{l}1970- \\
1975\end{array}$ & $\mathrm{RCT}$ & 19 & $\begin{array}{l}\text { Cardiac death (death } \\
\text { due to heart failure, } \\
\text { myocardial infarct, or } \\
\text { coronary thrombosis) }\end{array}$ & Did not specify & $\begin{array}{l}\text { Yes for mastectomy + RT vs. } \\
\text { mastectomy alone }(R R=1.52 \\
95 \% \mathrm{Cl}=1.01-2.29 ; p=0.04)\end{array}$ \\
\hline \multirow[t]{2}{*}{$\begin{array}{l}\text { Rutqvist } \\
(1992)^{70}\end{array}$} & 960 & Sweden & $\begin{array}{l}1971- \\
1976\end{array}$ & $\mathrm{RCT}$ & 16 & Death due to IHD & ICD & $\begin{array}{l}\text { Yes for IHD mortality in } \\
\text { highest dose-volume RT } \\
\text { patients (HR=3.2, } p<0.05 \text { ). } \\
\text { No for lower dose-volume } \\
\text { patients }\end{array}$ \\
\hline & & & & & & & & $\begin{array}{l}\text { Note: High dose-volume was } \\
\text { defined as using left-sided } \\
\text { tangential 60Co fields. Low } \\
\text { dose-volume was defined as } \\
\text { using right-sided tangential } \\
60 \text { Co fields. Intermediate } \\
\text { dose was defined as using } \\
\text { electron techniques }\end{array}$ \\
\hline $\begin{array}{l}\text { Haybittle } \\
(1989)^{32}\end{array}$ & 2800 & $\begin{array}{l}\text { United } \\
\text { Kingdom }\end{array}$ & $\begin{array}{l}1970- \\
1975\end{array}$ & $\mathrm{RCT}$ & $13.2-18$ & $\begin{array}{l}\text { Cardiac deaths } \\
\text { (deaths due to heart } \\
\text { failure, Ml, coronary } \\
\text { thrombosis, and } \\
\text { other cardiovascular } \\
\text { deaths) }\end{array}$ & $\begin{array}{l}\text { Death report } \\
\text { forms, copies } \\
\text { of death } \\
\text { certificates, and } \\
\text { correspondence } \\
\text { with treating } \\
\text { clinicians and } \\
\text { examination of the } \\
\text { patient's notes }\end{array}$ & $\begin{array}{l}\text { Yes }(R R=1.65,95 \% C l= \\
1.05-2.58 ; p=0.03)\end{array}$ \\
\hline Jones $(1989)^{71}$ & 1461 & $\begin{array}{l}\text { United } \\
\text { Kingdom }\end{array}$ & $\begin{array}{l}1949- \\
1955\end{array}$ & $\mathrm{RCT}$ & $\begin{array}{l}\text { Did not } \\
\text { specify (up } \\
\text { to } 34 \text { years' } \\
\text { follow-up) }\end{array}$ & Cardiovascular death & Chart review & $\begin{array}{l}\text { Yes }(p=0.03) \text {, especially after } \\
15+\text { years of follow-up } \\
(p=0.0045)\end{array}$ \\
\hline Høst (1986) ${ }^{72}$ & 1115 & Norway & $\begin{array}{l}1964- \\
1972\end{array}$ & $\mathrm{RCT}$ & $11.0-20.0$ & Cardiac deaths (AMI) & Death certificates & Yes $(p=0.004)$ \\
\hline
\end{tabular}

A systematic review conducted by Drost et $\mathrm{al}^{81}$ found that the mean heart dose steadily decreased from $4.6 \mathrm{~Gy}$ in 2014 to $2.6 \mathrm{~Gy}$ in 2017 ( $\mathrm{p}=0.003$ ). Combining this with the dose-dependent relationship between major cardiac events and mean dose to the heart by Darby et al, ${ }^{40}$ it is likely that the decrease in mean heart dose owing to improved contemporary RT techniques has led to improved outcomes in breast cancer patients in recent years. Our findings are also in support of this hypothesis since all studies that found a significant association between RT laterality and CVM included treatment groups that started prior to 1985 . This is consistent with the systematic review by Cheng et al ${ }^{4}$ which found an increased risk of cardiovascular death and coronary heart disease associated with RT among studies in which the breast cancer patients were diagnosed and irradiated before $1980(\mathrm{RR}=1.45,95 \% \mathrm{CI}=1.14-1.89)$ compared with women diagnosed and irradiated after $1980(\mathrm{RR}=1.15,95 \% \mathrm{CI}=0.92-1.44 ; \mathrm{p}=0.04)$. Similarly, Giordano et $\mathrm{al}^{23}$ found that in 1979, the HR for ischaemic heart disease mortality in left-sided compared with rightsided disease was 1.50 (95\% CI=1.19-1.87), but this HR declined by $6 \%$ with each succeeding year between 1979 and $1988(\mathrm{HR}=0.94 ; 95 \% \mathrm{CI}=0.91-0.98)$.

As such, newer research has started to evaluate whether the use of modern linear accelerator machines instead of ${ }^{60} \mathrm{Co}$ fields and various contemporary radiation techniques reduces the cardiac radiation dose and subsequent cardiac toxicities. One example is intensitymodulated RT, which allows a more conformal target coverage without exposing organs at risk to as much radiation. ${ }^{82}$ Other notable advancements in cardiac sparing techniques include the use of deep inspiration 
Table 4. Studies assessing the risk of cardiovascular events in patients who received RT compared with those who did not receive RT.

\begin{tabular}{|c|c|c|c|c|c|c|c|c|}
\hline Study & $\begin{array}{l}\text { Study } \\
\text { population }\end{array}$ & Country & $\begin{array}{l}\text { Treat- } \\
\text { ment } \\
\text { period }\end{array}$ & Study design & $\begin{array}{l}\text { Median or } \\
\text { mean follow- } \\
\text { up time, y }\end{array}$ & Endpoint(s) & Endpoint criteria & $\begin{array}{l}\text { Significant increased risk of } \\
\text { cardiovascular events }\end{array}$ \\
\hline $\begin{array}{l}\text { Chou } \\
(2020)^{59}\end{array}$ & 27,466 & Taiwan & $\begin{array}{l}2007- \\
2014\end{array}$ & $\begin{array}{l}\text { Population- } \\
\text { based cohort }\end{array}$ & $\begin{array}{l}\text { Did not } \\
\text { specify }\end{array}$ & $\begin{array}{l}\text { MACE, identified by } \\
\text { emergency visit claims } \\
\text { or inpatient data of IHD, } \\
\mathrm{CHF} \text {, acute ischaemic } \\
\text { stroke, and intracranial } \\
\text { haemorrhage }\end{array}$ & $\begin{array}{l}\text { ICD-9 Clinical } \\
\text { Modification }\end{array}$ & $\begin{array}{l}\text { No, } \mathrm{RT}+\text { hormone group } \\
\text { vs. hormone only group } \\
\text { (HR=0.831, 95\% Cl= } \\
0.593-1.164 ; p=0.286)\end{array}$ \\
\hline $\begin{array}{l}\text { Killander } \\
(2020)^{54}\end{array}$ & 1144 & Sweden & $\begin{array}{l}1991- \\
1997\end{array}$ & RCT & 21.3 & $\begin{array}{l}\text { Cardiac morbidity and } \\
\text { mortality }\end{array}$ & $\begin{array}{l}\text { ICD-8, ICD-9, } \\
\text { ICD-10 }\end{array}$ & $\begin{array}{l}\text { No, incidence of admission } \\
\text { to hospital with a cardiac } \\
\text { diagnosis was } 29.7 \% \text { in no } \\
\text { RT group, vs. } 31 \% \text { in RT } \\
\text { group ( } p=0.7 \text { ) }\end{array}$ \\
\hline $\begin{array}{l}\text { Hamood } \\
(2019)^{73}\end{array}$ & 338 & Israel & $\begin{array}{l}2002- \\
2012\end{array}$ & $\begin{array}{l}\text { Population- } \\
\text { based cohort }\end{array}$ & 5.7 & $\begin{array}{l}\text { CVD events (IHD, } \\
\text { CHF, arrhythmias and } \\
\text { conduction disorders) }\end{array}$ & ICD-9 & $\begin{array}{l}\text { Yes (HR=2.94, } \\
95 \% \mathrm{Cl}=1.17-7.38 ; p=0.22)\end{array}$ \\
\hline $\begin{array}{l}\text { Jacobse } \\
(2019)^{74}\end{array}$ & $\begin{array}{l}365 \text { (183 } \\
\text { Ml cases } \\
\text { and } 182 \\
\text { controls) }\end{array}$ & Netherlands & $\begin{array}{l}1970- \\
2009\end{array}$ & $\begin{array}{l}\text { Population- } \\
\text { based } \\
\text { case-control }\end{array}$ & $\begin{array}{l}13.6 \text { years } \\
\text { median time } \\
\text { to } \mathrm{Ml}\end{array}$ & $\mathrm{Ml}$ & Did not specify & $\begin{array}{l}\text { Yes for } \mathrm{RT} \text { with } \mathrm{IMC} \\
(\mathrm{RR}=2.45,95 \% \mathrm{Cl}= \\
1.97-3.05 ; \mathrm{p}=0.006) \text {, } \\
\text { No for } \mathrm{RT} \text { without IMC } \\
(\mathrm{RR}=1.20,95 \% \mathrm{Cl}= \\
0.67-2.18)\end{array}$ \\
\hline $\begin{array}{l}\text { Lawrenson } \\
(2019)^{61}\end{array}$ & 3528 & $\begin{array}{l}\text { New } \\
\text { Zealand }\end{array}$ & $\begin{array}{l}1995- \\
2013\end{array}$ & $\begin{array}{l}\text { Population- } \\
\text { based cohort }\end{array}$ & $\begin{array}{l}\text { 10-year } \\
\text { follow-up } \\
\text { period }\end{array}$ & $\begin{array}{l}\text { Cardiovascular events } \\
\text { and cardiovascular } \\
\text { mortality }\end{array}$ & ICD-9 & $\begin{array}{l}\text { No }(\mathrm{HR}=0.73,95 \% \mathrm{Cl}= \\
0.59-0.92)\end{array}$ \\
\hline $\begin{array}{l}\text { Lee } \\
(2019)^{75}\end{array}$ & 1759 & Taiwan & $\begin{array}{l}2002- \\
2012\end{array}$ & $\begin{array}{l}\text { Population- } \\
\text { based cohort }\end{array}$ & 5.2 & $\begin{array}{l}\text { Major heart events (heart } \\
\text { failure and CAD) }\end{array}$ & ICD-9 & $\begin{array}{l}\text { Yes }(H R=1.47 \\
95 \% \mathrm{Cl}=1.24-1.73 \\
p<0.0001)\end{array}$ \\
\hline $\begin{array}{l}\text { Wadsten } \\
(2018)^{44}\end{array}$ & 6270 & Sweden & $\begin{array}{l}1992- \\
2012\end{array}$ & $\begin{array}{l}\text { Population- } \\
\text { based cohort }\end{array}$ & 8.8 & $\mathrm{IHD}$ & ICD-9, ICD-10 & $\begin{array}{l}\text { No }(H R=0.79,95 \% \mathrm{Cl}= \\
0.62-1.01)\end{array}$ \\
\hline Wu $(2017)^{76}$ & 746 & $\begin{array}{l}\text { United } \\
\text { States }\end{array}$ & $\begin{array}{l}1997- \\
1999\end{array}$ & $\mathrm{RCT}$ & 10.5 & $\begin{array}{l}\text { Cardiac adverse event } \\
\text { (IHD, MI, heart failure, } \\
\text { relative and absolute } \\
\text { decrease of LVEF > } 20 \% \\
\text { from baseline, and } \\
\text { incidence of arrhythmia) }\end{array}$ & $\begin{array}{l}\text { National Cancer } \\
\text { Institute common } \\
\text { toxicity criteria for } \\
\text { adverse events }\end{array}$ & $\begin{array}{l}\text { No }(7.9 \% \text { for RT vs. } 8 \% \text { for } \\
\text { no RT }[p=1.0])\end{array}$ \\
\hline $\begin{array}{l}\text { Boekel } \\
(2016)^{36}\end{array}$ & 59,388 & Netherlands & $\begin{array}{l}1989- \\
2005\end{array}$ & $\begin{array}{l}\text { Population- } \\
\text { based cohort }\end{array}$ & 9 & $\begin{array}{l}\text { Cardiovascular event } \\
\text { (a cardiovascular } \\
\text { hospital discharge } \\
\text { diagnosis, cardiosurgical } \\
\text { intervention, or death due } \\
\text { to CVD) }\end{array}$ & ICD-10 & $\begin{array}{l}\text { Yes for left-sided RT after } \\
\text { mastectomy vs. surgery } \\
\text { alone (subdistribution } \\
\mathrm{HR}=1.23,95 \% \mathrm{Cl}= \\
1.11-1.36 \text { ) }\end{array}$ \\
\hline $\begin{array}{l}\text { Leung } \\
(2016)^{62}\end{array}$ & 5132 & Taiwan & $\begin{array}{l}2000- \\
2010\end{array}$ & $\begin{array}{l}\text { Population- } \\
\text { based } \\
\text { case-control } \\
\text { study }\end{array}$ & 3.5 & $\begin{array}{l}\text { IHD, valvular heart } \\
\text { disease, CHF, and/or } \\
\text { conduction abnormalities }\end{array}$ & ICD-9 & $\begin{array}{l}\text { No }(H R=0.81,95 \% \mathrm{Cl}= \\
0.57-1.15)\end{array}$ \\
\hline $\begin{array}{l}\operatorname{Tan} \\
(2016)^{77}\end{array}$ & 5514 & Taiwan & $\begin{array}{l}2002- \\
2007\end{array}$ & $\begin{array}{l}\text { Population- } \\
\text { based cohort }\end{array}$ & 5.3 & CVD (IHD and CHF) & ICD-9 & $\begin{array}{l}\text { No for } R T \text { vs. surgery alone } \\
(H R=0.97,95 \% \mathrm{Cl}= \\
0.62-1.51 ; p=0.882) \\
\text { Yes for Chemo + RT vs. } \\
\text { surgery alone }(H R=1.84 \text {, } \\
95 \% \mathrm{Cl}=1.34-2.53 ; p<0.001)\end{array}$ \\
\hline $\begin{array}{l}\text { Boekel } \\
(2014)^{7}\end{array}$ & $\begin{array}{l}10,365 \\
\text { (DCIS } \\
\text { patients } \\
\text { only) }\end{array}$ & Netherlands & $\begin{array}{l}1989- \\
2004\end{array}$ & $\begin{array}{l}\text { Population- } \\
\text { based cohort }\end{array}$ & 10 & $\begin{array}{l}\text { MI, other IHDs, } \\
\text { other heart disease, } \\
\text { pericarditis, valvular } \\
\text { dysfunction, } \\
\text { cardiomyopathy, } \\
\text { arrhythmia, CHF, and/or } \\
\text { cerebrovascular disease }\end{array}$ & ICD-10 & $\begin{array}{l}\text { No for patients from } \\
1997-2005 \text { who received } \\
\text { left-sided } \mathrm{RT}(\mathrm{HR}=0.96 \text {, } \\
95 \% \mathrm{Cl}=0.75-1.23 \text { ) or } \\
\text { right-sided } \mathrm{RT}(\mathrm{HR}=1.02 \text {, } \\
95 \% \mathrm{Cl}=0.78-1.33) \text { vs. } \\
\text { surgery only } \\
\text { No for the entire study } \\
\text { population who received } \\
\text { left-sided } \mathrm{RT}(\mathrm{HR}=1.01 \text {, } \\
\text { 95\% } \mathrm{Cl}=0.79-1.30) \text { or } \\
\text { right-sided } \mathrm{RT}(\mathrm{HR}=1.08 \text {, } \\
\text { 95\% } \mathrm{Cl}=0.83-1.41) \text { vs. } \\
\text { surgery only }\end{array}$ \\
\hline
\end{tabular}

Abbreviations: 95\% Cl = confidence interval; $\mathrm{AMI}$ = acute myocardial infarction; BCS = breast-conserving surgery; CAD = coronary artery disease; $\mathrm{CHF}=$ congestive heart failure; $\mathrm{CVD}=$ cardiovascular diseases; $\mathrm{DCIS}=$ ductal carcinoma in situ; $\mathrm{HR}=$ hazard ratio; $I C D=$ International Classification of Diseases; ICPC = International Classification of Primary Care; IHD = ischaemic heart disease; IMC = internal mammary chain; LVEF = left ventricular ejection fraction; MACE = major adverse cardiac events; $\mathrm{Ml}=$ myocardial infarction; OR = odds ratio; $\mathrm{RCT}$ = randomised controlled trial; $\mathrm{RR}$ = risk ratio; $\mathrm{RT}$ = radiotherapy. 
Table 4. (cont'd)

\begin{tabular}{|c|c|c|c|c|c|c|c|c|}
\hline Study & $\begin{array}{l}\text { Study } \\
\text { population }\end{array}$ & Country & $\begin{array}{l}\text { Treat- } \\
\text { ment } \\
\text { period }\end{array}$ & Study design & $\begin{array}{l}\text { Median or } \\
\text { mean follow- } \\
\text { up time, y }\end{array}$ & Endpoint(s) & Endpoint criteria & $\begin{array}{l}\text { Significant increased risk of } \\
\text { cardiovascular events }\end{array}$ \\
\hline $\begin{array}{l}\text { Boerman } \\
(2014)^{45}\end{array}$ & 456 & Netherlands & $\begin{array}{l}1970- \\
2007\end{array}$ & $\begin{array}{l}\text { Matched case- } \\
\text { control study }\end{array}$ & 9 & $\begin{array}{l}\text { CHF (acute and chronic), } \\
\text { vascular cardiac } \\
\text { diseases (unstable and } \\
\text { stable angina pectoris, } \\
\text { AMl, other chronic } \\
\text { IHDs [coronary artery } \\
\text { sclerosis], transient } \\
\text { ischaemic attack, and } \\
\text { cerebrovascular attack), } \\
\text { and other cardiac } \\
\text { diseases (atrial fibrillation, } \\
\text { paroxysmal tachycardia, } \\
\text { non-rheumatic valve } \\
\text { disease) }\end{array}$ & ICPC version 1 & $\begin{array}{l}\text { No for } \mathrm{CHF}(\mathrm{HR}=0.4 \text {, } \\
95 \% \mathrm{Cl}=0.2-1.1) \text {, vascular } \\
\text { cardiac diseases }(\mathrm{HR}=0.7 \text {, } \\
95 \% \mathrm{Cl}=0.4-1.1) \text { and other } \\
\text { cardiac diseases }(\mathrm{HR}=0.9 \text {, } \\
95 \% \mathrm{Cl}=0.5-1.6)\end{array}$ \\
\hline $\begin{array}{l}\text { Killander } \\
(2014)^{63}\end{array}$ & 1110 & Sweden & $\begin{array}{l}1978- \\
1985\end{array}$ & Two RCTs & Not specified & $\begin{array}{l}\text { Incidence of first } \\
\text { admission to hospital due } \\
\text { to heart disease }\end{array}$ & $\begin{array}{l}\text { ICD-8, ICD-9, } \\
\text { ICD-10 }\end{array}$ & $\begin{array}{l}\text { No, when RT + } \\
\text { cyclophosphamide } \\
\text { was compared with } \\
\text { cyclophosphamide alone, } \\
\text { in premenopausal women } \\
(p=0.72) \text { or when } R T+ \\
\text { tamoxifen was compared } \\
\text { with tamoxifen alone in } \\
\text { postmenopausal women } \\
(p=0.41)\end{array}$ \\
\hline \multirow[t]{2}{*}{$\begin{array}{l}\text { Onwudiwe } \\
(2014)^{78}\end{array}$} & \multirow[t]{2}{*}{91,612} & \multirow[t]{2}{*}{$\begin{array}{l}\text { United } \\
\text { States }\end{array}$} & \multirow[t]{2}{*}{$\begin{array}{l}2000- \\
2005\end{array}$} & \multirow[t]{2}{*}{$\begin{array}{l}\text { Population- } \\
\text { based } \\
\text { case-control }\end{array}$} & \multirow[t]{2}{*}{2} & \multirow{2}{*}{$\begin{array}{l}\text { Combined risk of } \\
\text { cardiovascular event } \\
\text { (angina, heart failure, } \\
\text { chest pain, ischaemia, } \\
\text { valve disorder, heart } \\
\text { disease, atherosclerosis, } \\
\text { cardiac inflammation, } \\
\text { conduction disorder, } \\
\text { Ml, cardiomyopathy, or } \\
\text { elevated blood pressure) } \\
\text { or cardiovascular death } \\
\text { within } 6 \text { months of RT }\end{array}$} & \multirow[t]{2}{*}{ ICD-9 } & $\begin{array}{l}\text { Yes for high-risk patients } \\
\text { (no RT vs. RT, HR=0.723, } \\
95 \% \mathrm{Cl}=0.685-0.763 \text { ) and } \\
\text { intermediate-risk patients (no } \\
\text { RT vs. RT, HR=0.746, } \\
95 \% \mathrm{Cl}=0.650-0.857 \text { ) } \\
\text { No for low-risk patients (no } \\
\text { RT vs. RT, HR=1.007, } \\
95 \% \mathrm{Cl}=0.838-1.211 \text { ) }\end{array}$ \\
\hline & & & & & & & & $\begin{array}{l}\text { Note: High-risk patients were } \\
\text { defined as patients with a } \\
\text { clinical CVD. Intermediate-risk } \\
\text { patients were defined as } \\
\text { patients with one or more risk } \\
\text { factors and no diagnosis of } \\
\text { CVD. Low-risk patients were } \\
\text { defined as patients who did } \\
\text { not meet the criteria for high } \\
\text { or intermediate risk }\end{array}$ \\
\hline $\begin{array}{l}\text { Haque } \\
(2011)^{49}\end{array}$ & 806 & $\begin{array}{l}\text { United } \\
\text { States }\end{array}$ & $\begin{array}{l}1990- \\
1994\end{array}$ & $\begin{array}{l}\text { Population- } \\
\text { based cohort }\end{array}$ & 10 & $\begin{array}{l}\text { CVD events that were } \\
\text { serious enough to require } \\
\text { hospitalisation IHD, AMI, } \\
\text { angina, and CVD) }\end{array}$ & ICD-9 and ICD-10 & $\begin{array}{l}\text { No for left-sided } \mathrm{RT} \text { vs. no RT } \\
(\mathrm{HR}=1.00,95 \% \mathrm{Cl}= \\
0.75-1.35) \text { and for right-sided } \\
\mathrm{RT} \text { vs. no } \mathrm{RT} \text { (HR=0.75, } \\
95 \% \mathrm{Cl}=0.53-1.06)\end{array}$ \\
\hline $\begin{array}{l}\text { Doyle } \\
(2007)^{48}\end{array}$ & 48,353 & $\begin{array}{l}\text { United } \\
\text { States }\end{array}$ & $\begin{array}{l}1992- \\
2000\end{array}$ & $\begin{array}{l}\text { Population- } \\
\text { based cohort }\end{array}$ & 13 as max & $\begin{array}{l}\text { MI, ischaemia, CHF, } \\
\text { and other heart disease } \\
\text { (myocarditis, arrhythmia, } \\
\text { and valvular disease) }\end{array}$ & ICD-9 & $\begin{array}{l}\text { No for } \mathrm{Ml} / \text { ischaemia } \\
(\mathrm{HR}=1.02,95 \% \mathrm{Cl}= \\
0.94-1.10) \text { or for } \mathrm{Ml} \text { alone } \\
(\mathrm{HR}=0.93,95 \% \mathrm{Cl}= \\
0.84-1.02)\end{array}$ \\
\hline $\begin{array}{l}\text { Hooning } \\
(2007)^{79}\end{array}$ & 4414 & Netherlands & $\begin{array}{l}1970- \\
1986\end{array}$ & $\begin{array}{l}\text { Multicentre } \\
\text { case-control } \\
\text { study }\end{array}$ & 17.7 & $\begin{array}{l}\text { IHD (AMl, angina), other } \\
\text { heart diseases (pericarditis, } \\
\text { valvular dysfunction, } \\
\text { cardiomyopathy, } \\
\text { dysrhythmia, CHF), and/or } \\
\text { other CVDs }\end{array}$ & ICD-9 & $\begin{array}{l}\text { Yes for CVDs (HR=1.41, } \\
95 \% \mathrm{Cl}=1.14-1.74) .1 .49 \text {-fold } \\
\text { increased risk for RT patients } \\
\text { during a period of } 1970-1979 \\
\text { and } 1.35 \text {-fold increased risk } \\
\text { during a period of } 1980-1986\end{array}$ \\
\hline $\begin{array}{l}\text { Geiger } \\
(2005)^{80}\end{array}$ & $\begin{array}{l}396 \text { ( } 134 \\
\text { cases and } \\
262 \text { controls) }\end{array}$ & $\begin{array}{l}\text { United } \\
\text { States }\end{array}$ & $\begin{array}{l}1980- \\
2000\end{array}$ & Case-control & $\begin{array}{l}\text { Did not } \\
\text { specify }\end{array}$ & $\mathrm{Ml}$ & ICD-9, ICD-10 & $\begin{array}{l}\text { Yes }(\mathrm{OR}=2.0,95 \% \mathrm{Cl}= \\
1.1-3.5)\end{array}$ \\
\hline $\begin{array}{l}\text { Højris } \\
(1999)^{67}\end{array}$ & 3046 & Denmark & $\begin{array}{l}1982- \\
1990\end{array}$ & Two RCTs & 9.75 & IHD (including AMI) & ICD-8, ICD-10 & $\begin{array}{l}\text { No }(H R=0.95,95 \% \mathrm{Cl}= \\
0.59-1.54)\end{array}$ \\
\hline $\begin{array}{l}\text { Gyenes } \\
(1998)^{68}\end{array}$ & 960 & Sweden & $\begin{array}{l}1971- \\
1976\end{array}$ & $\begin{array}{l}\text { Multicentre } \\
\text { case-control } \\
\text { study }\end{array}$ & 20 & IHD and/or CVD & ICD-9 & $\begin{array}{l}\text { No }(H R=1.3,95 \% \mathrm{Cl}= \\
0.7-2.6)\end{array}$ \\
\hline $\begin{array}{l}\text { Rutqvist } \\
(1998)^{69}\end{array}$ & 5680 & Sweden & $\begin{array}{l}1976- \\
1987\end{array}$ & $\begin{array}{l}\text { Hospital-based } \\
\text { case-cohort }\end{array}$ & 9 & $\mathrm{AMl}$ & Did not specify & $\begin{array}{l}\text { No for } \mathrm{RT}+\mathrm{BCS} \text { vs. } \\
\text { mastectomy alone }(\mathrm{HR}=0.6 \text {, } \\
95 \% \mathrm{Cl}=0.4-1.2)\end{array}$ \\
\hline
\end{tabular}


breath hold, enhanced patient positioning, and heart blocking. ${ }^{83}$ Deep inspiration breath hold and respiratory gating rely on the principle that during inspiration, the diaphragm flattens, and the lungs expand, causing the heart to be pulled away from the chest wall and thus decrease the radiation dose to the heart and the left anterior descending artery. ${ }^{84}$ A 2019 study conducted by Simonetto et $\mathrm{al}^{85}$ found that the use of deep inspiration breath hold reduced the risk of estimated mean heart dose by $35 \%$, compared with free breathing. Furthermore, to reduce the heart dose in breast cancer patients, prone positioning can be used to increase the planning target volume to heart distance by displacing cardiac structures and substructures out of irradiated volumes. ${ }^{86-88}$ Other common methods include multileaf collimator modification during RT planning. ${ }^{89,90}$ However, an important pitfall is that it may shield part of the breast tissue, which needs to be irradiated; thus, a balance must be achieved in order to maximise the heart shielding while minimising the target volume missed. ${ }^{89}$ In addition to these RT techniques, the omission of internal mammary chain lymph node irradiation and rib inclusion for chest wall RT has been utilised in earlystage breast cancer patients to reduce the dose to the normal tissue. However, long-term studies are needed to investigate the effect of contemporary RT planning techniques in minimising radiation exposure to nearby normal tissue and the heart.

In addition to the use of modern RT techniques, considerations must be made in terms of whether $\mathrm{RT}$ is being combined with chemotherapeutic agents as common chemotherapeutic agents have known cardiotoxic effects. ${ }^{91-94}$ A 10-year cohort study of breast cancer patients receiving concomitant RT and chemotherapy found that there was no significant association between CVEs and RT laterality (HR=2.38, 95\% CI $=0.80-7.11 ; \mathrm{p}=0.12) .{ }^{55}$ However, there was a significant increase in CVEs for patients receiving left-sided RT with a doxorubicin-equivalent dose $\geq 250 \mathrm{mg} / \mathrm{m}^{2}$ compared with patients receiving rightsided RT with a cumulative doxorubicin-equivalent dose $<250 \mathrm{mg} / \mathrm{m}^{2}(\mathrm{HR}=5.22,95 \% \mathrm{CI}=1.67-21.15$; $\mathrm{p}=0.006) .{ }^{55}$ Similarly, a 2016 study found that there was no significant increase in the risk of CVEs for patients who received RT compared with those who received surgery alone $(\mathrm{HR}=0.97,95 \% \mathrm{CI}=0.62-1.51 ; \mathrm{p}=0.882) .{ }^{77}$ However, there was a significant increase in CVEs in patients who received RT and chemotherapy compared with those who received surgery only $(\mathrm{HR}=1.84$; $95 \% \mathrm{CI}=1.34-2.53 ; \mathrm{p}<0.001){ }^{77}$
There are several limitations to this systematic review. First, the heterogeneity of the data made it difficult to make direct comparisons among studies. Many of the studies did not report data on the individual types of CVEs or the specific cause of CVM, and so we used a composite outcome of CVE and CVM, which included myocardial infarction, coronary artery disease, conduction abnormalities, congestive heart failure, and other cardiovascular diseases. Heterogeneity also exists because of the lack of detail on RT techniques, RT volume, dose, and fractionation. In addition, variability in morbidity and mortality assessment, as well as in the follow-up time of the studies, is another source of heterogeneity. Second, the dose-dependent relationship of cardiovascular risk cannot be evaluated since radiation doses were not available for all studies. Lastly, because this was a systematic review, and a meta-analysis was not conducted, studies were not weighted based on the number of patients. In the future, a meta-analysis would aid in determining whether there is a significant increase in the risk of CVEs and CVM associated with the use of RT and/or RT laterality. Other confounding variables may also be investigated and stratified, such as menopausal status, types of adjuvant chemotherapy and hormonal agents, which may impact cardiotoxicity.

\section{CONCLUSION}

Although modern RT techniques seem to have minimised the cardiac exposure in breast cancer patients receiving $\mathrm{RT}$, more comprehensive studies with longer follow-up periods must be conducted to investigate any associated cardiovascular risk.

\section{REFERENCES}

1. Bray F, Ferlay J, Soerjomataram I, Siegel RL, Torre LA, Jemal A. Global cancer statistics 2018: GLOBOCAN estimates of incidence and mortality worldwide for 36 cancers in 185 countries. CA Cancer J Clin. 2018;68:394-424.

2. EBCTCG (Early Breast Cancer Trialists' Collaborative Group); McGale P, Taylor C, Correa C, Cutter D, Duane F, et al. Effect of radiotherapy after mastectomy and axillary surgery on 10-year recurrence and 20-year breast cancer mortality: meta-analysis of individual patient data for 8135 women in 22 randomised trials. Lancet. 2014;383:2127-35.

3. Early Breast Cancer Trialists' Collaborative Group (EBCTCG), Darby S, McGale P, Correa C, Taylor C, Arriagada R, et al. Effect of radiotherapy after breast-conserving surgery on 10-year recurrence and 15-year breast cancer death: meta-analysis of individual patient data for 10,801 women in 17 randomised trials. Lancet. 2011;378:1707-16.

4. Cheng YJ, Nie XY, Ji CC, Lin XX, Liu LJ, Chen XM, et al. Longterm cardiovascular risk after radiotherapy in women with breast cancer. J Am Heart Assoc. 2017;6:e005633.

5. Henson KE, McGale P, Darby SC, Parkin M, Wang Y, Taylor CW. Cardiac mortality after radiotherapy, chemotherapy and endocrine 
therapy for breast cancer: cohort study of 2 million women from 57 cancer registries in 22 countries. Int J Cancer. 2020;147:1437-49.

6. Beaton L, Bergman A, Nichol A, Aparicio M, Wong G, Gondara L, et al. Cardiac death after breast radiotherapy and the QUANTEC cardiac guidelines. Clin Transl Radiat Oncol. 2019;19:39-45.

7. Boekel NB, Schaapveld M, Gietema JA, Rutgers EJ, Versteegh MI, Visser O, et al. Cardiovascular morbidity and mortality after treatment for ductal carcinoma in situ of the breast. J Natl Cancer Inst. 2014;106:dju156.

8. Henson KE, McGale P, Taylor C, Darby SC. Radiation-related mortality from heart disease and lung cancer more than 20 years after radiotherapy for breast cancer. Br J Cancer. 2013;108:179-82 .

9. Tjessem KH, Johansen S, Malinen E, Reinertsen KV, Danielsen T, Fosså SD, et al. Long-term cardiac mortality after hypofractionated radiation therapy in breast cancer. Int J Radiat Oncol Biol Phys. 2013;87:337-43.

10. Bouillon K, Haddy N, Delaloge S, Garbay JR, Garsi JP, Brindel P, et al. Long-term cardiovascular mortality after radiotherapy for breast cancer. J Am Coll Cardiol. 2011;57:445-52.

11. McGale P, Darby SC, Hall P, Adolfsson J, Bengtsson NO, Bennet AM, et al. Incidence of heart disease in 35,000 women treated with radiotherapy for breast cancer in Denmark and Sweden. Radiother Oncol. 2011;100:167-75.

12. Park CK, Li X, Starr J, Harris EE. Cardiac morbidity and mortality in women with ductal carcinoma in situ of the breast treated with breast conservation therapy. Breast J. 2011;17:470-6.

13. Stokes EL, Tyldesley S, Woods R, Wai E, Olivotto IA. Effect of nodal irradiation and fraction size on cardiac and cerebrovascular mortality in women with breast cancer treated with local and locoregional radiotherapy. Int J Radiat Oncol Biol Phys. 2011;80:403-9.

14. Wang W, O'Connell D, Stuart K, Boyages J. Analysis of 10-year cause-specific mortality of patients with breast cancer treated in New South Wales in 1995. J Med Imaging Radiat Oncol. 2011;55:516-25.

15. Bouchardy C,Rapiti E, Usel M, Majno SB, Vlastos G, Benhamou S, et al. Excess of cardiovascular mortality among node-negative breast cancer patients irradiated for inner-quadrant tumors. Ann Oncol. 2010;21:459-65.

16. Gutt R, Correa CR, Hwang WT, Solin LJ, Litt HI, Ferrari VA, et al. Cardiac morbidity and mortality after breast conservation treatment in patients with early-stage breast cancer and preexisting cardiac disease. Clin. Breast Cancer. 2008;8:443-8.

17. Li WH, Zhang ZG, Huang ZR, Zhang W, Li ZB, Qi ZQ. No association between tumor laterality and cardiac-related mortality in breast cancer patients after radiotherapy: a population-based study. Cancer Manag Res. 2018;10:3649-56.

18. Borger JH, Hooning MJ, Boersma LJ, Snijders-Keilholz A, Aleman BM, Lintzen E, et al. Cardiotoxic effects of tangential breast irradiation in early breast cancer patients: the role of irradiated heart volume. Int J Radiat Oncol Biol Phys. 2007;69:1131-8.

19. Marhin W, Wai E, Tyldesley S. Impact of fraction size on cardiac mortality in women treated with tangential radiotherapy for localized breast cancer. Int J Radiat Oncol Biol Phys. 2007;69:4839.

20. Paszat LF, Vallis KA, Benk VM, Groome PA, Mackillop WJ, Wielgosz A. A population-based case-cohort study of the risk of myocardial infarction following radiation therapy for breast cancer. Radiother Oncol. 2007;82:294-300.

21. Roychoudhuri R, Robinson D, Putcha V, Cuzick J, Darby S, Møller H. Increased cardiovascular mortality more than fifteen years after radiotherapy for breast cancer: a population-based study. BMC Cancer. 2007;7:9.
22. Harris EE, Correa C, Hwang WT, Liao J, Litt HI, Ferrari VA, et al. Late cardiac mortality and morbidity in early-stage breast cancer patients after breast-conservation treatment. J Clin Oncol. 2006;24:4100-6.

23. Giordano SH, Kuo YF, Freeman JL, Buchholz TA, Hortobagyi GN, Goodwin JS. Risk of cardiac death after adjuvant radiotherapy for breast cancer. J Natl Cancer Inst. 2005;97:419-24.

24. Vallis KA, Pintilie M, Chong N, Holowaty E, Douglas PS, Kirkbride $\mathrm{P}$, et al. Assessment of coronary heart disease morbidity and mortality after radiation therapy for early breast cancer. J Clin Oncol. 2002;20:1036-42.

25. Nixon AJ, Manola J, Gelman R, Bornstein B, Abner A, Hetelekidis S, et al. No long-term increase in cardiac-related mortality after breastconserving surgery and radiation therapy using modern techniques. J Clin Oncol. 1998;16:1374-9.

26. Paszat LF, Mackillop WJ, Groome PA, Schulze K, Holowaty E. Mortality from myocardial infarction following postlumpectomy radiotherapy for breast cancer: A population-based study in Ontario, Canada. Int J Radiat Oncol Biol Phys. 1999;43:755-62.

27. Obi N, Eulenburg C, Seibold P, Eilber U, Thöne K, Behrens S, et al. Associations between adjuvant radiotherapy and different causes of death in a German breast cancer cohort. Breast. 2018;38:75-80.

28. Paszat LF, Mackillop WJ, Groome PA, Boyd C, Schulze K, Holowaty E. Mortality from myocardial infarction after adjuvant radiotherapy for breast cancer in the surveillance, epidemiology, and end-results cancer registries. J Clin Oncol. 1998;16:2625-31 .

29. Cuzick J, Stewart H, Rutqvist L, Honghton J, Edwards R, Redmond $\mathrm{C}$, et al. Cause-specific mortality in long-term survivors of breast cancer who participated in trials of radiotherapy. J Clin Oncol. 1994;12:447-53.

30. Houghton J, Baum M, Haybittle JL. Role of radiotherapy following total mastectomy in patients with early breast cancer. World J Surg. 1994;18:117-22.

31. Rutqvist LE, Johansson H. Mortality by laterality of the primary tumour among 55,000 breast cancer patients from the Swedish cancer registry. Br J Cancer. 1990;61:866-8.

32. Haybittle JL, Brinkley D, Houghton J, A’Hern RP, Baum M. Postoperative radiotherapy and late mortality: evidence from the Cancer Research Campaign trial for early breast cancer. BMJ. 1989;298:1611-4.

33. Chang JS, Ko BK, Bae JH, Yu JH, Park MH, Jung Y, et al. Radiation-related heart disease after breast cancer radiation therapy in Korean women. Breast Cancer Res Treat. 2017;166:249-57.

34. Haque W, Verma V, Haque A,Butler EB, Teh BS. Trends in cardiac mortality in women with ductal carcinoma in situ. Breast Cancer Res Treat. 2017;161:345-51.

35. Merzenich H, Bartkowiak D, Schmidberger H, Schmidt M, Schwentner L, Wiegel T, et al. 3D conformal radiotherapy is not associated with the long-term cardiac mortality in breast cancer patients: a retrospective cohort study in Germany (PASSOS-Heart Study). Breast Cancer Res Treat. 2017;161:143-52.

36. Boekel NB, Schaapveld M, Gietema JA, Russell NS, Poortmans P, Theuws JC, et al. Cardiovascular disease risk in a large, populationbased cohort of breast cancer survivors. Int J Radiat Oncol Biol Phys. 2016;94:1061-72.

37. Paul Wright G, Drinane JJ, Sobel HL, Chung MH. Left-sided breast irradiation does not result in increased long-term cardiac-related mortality among women treated with breast-conserving surgery. Ann Surg Oncol. 2016;23:1117-22.

38. Ye JC, Yan W, Christos P, Nori D, Chao KS, Ravi A. Second cancer, breast cancer, and cardiac mortality in stage T1aNO breast cancer patients with or without external beam radiation therapy: a national registry study. Clin Breast Cancer. 2015;15:54-9. 
39. Darby SC, McGale P, Taylor CW, Peto R. Long-term mortality from heart disease and lung cancer after radiotherapy for early breast cancer: prospective cohort study of about 300000 women in US SEER cancer registries. Lancet Oncol. 2005;6:557-65.

40. Darby SC, Ewertz M, McGale P, Bennet AM, Blom-Goldman U, Brønnum D, et al. Risk of ischemic heart disease in women after radiotherapy for breast cancer. N Engl J Med. 2013;368:987-98.

41. Jagsi R, Griffith KA, Koelling T, Roberts R, Pierce LJ. Rates of myocardial infarction and coronary artery disease and risk factors in patients treated with radiation therapy for early-stage breast cancer. Cancer. 2007;109:650-7.

42. Patt DA, Goodwin JS, Kuo YF, Freeman JL, Zhang DD, Buchholz TA, et al. Cardiac morbidity of adjuvant radiotherapy for breast cancer. J Clin Oncol. 2005;23:7475-82.

43. Dess RT, Liss AL, Griffith KA, Marsh RB, Moran JM, Mayo C, et al. Ischemic cardiac events following treatment of the internal mammary nodal region using contemporary radiation planning techniques. Int J Radiat Oncol Biol Phys. 2017;99:1146-53.

44. Wadsten C, Wennstig AK, Garmo H, Nilsson G, Blomqvist C, Holmberg $\mathrm{L}$, et al. Risk of ischemic heart disease after radiotherapy for ductal carcinoma in situ. Breast Cancer Res Treat. 2018;171:95101.

45. Boerman LM, Berendsen AJ, van der Meer P, Maduro JH, Berger MY, de Bock GH. Long-term follow-up for cardiovascular disease after chemotherapy and/or radiotherapy for breast cancer in an unselected population. Support Care Cancer. 2014;22:1949-58.

46. Rehammar JC, Jensen MB, McGale P, Lorenzen EL, Taylor C, Darby SC, et al. Risk of heart disease in relation to radiotherapy and chemotherapy with anthracyclines among 19,464 breast cancer patients in Denmark, 1977-2005. Radiother Oncol. 2017;123:299305 .

47. Boekel NB, Jacobse JN, Schaapveld M, Hooning MJ, Gietema JA, Duane FK, et al. Cardiovascular disease incidence after internal mammary chain irradiation and anthracycline-based chemotherapy for breast cancer. Br J Cancer. 2018;119:408-18.

48. Doyle JJ, Neugut AI, Jacobson JS, Wang J, McBride R, Grann A, et al. Radiation therapy, cardiac risk factors, and cardiac toxicity in early-stage breast cancer patients. Int J Radiat Oncol Biol Phys. 2007;68:82-93.

49. Haque R, Yood MU, Geiger AM, Kamineni A, Avila CC, Shi J, et al. Long-term safety of radiotherapy and breast cancer laterality in older survivors. Cancer Epidemiol Biomarkers Prev. 2011;20:2120-6.

50. Soran O, Vargo JA, Polat AV, Soran A, Sumkin J, Beriwal S. No association between left-breast radiation therapy or breast arterial calcification and long-term cardiac events in patients with breast cancer. J Womens Health (Larchmt). 2014;23:1005-11.

51. Abouegylah M, Braunstein LZ, Alm El-Din MA, Niemierko A, Salama L, Elebrashi M, et al. Evaluation of radiation-induced cardiac toxicity in breast cancer patients treated with trastuzumabbased chemotherapy. Breast Cancer Res Treat. 2019;174:179-85.

52. Chang JS, Shin J, Park EC, Kim YB. Risk of cardiac disease after adjuvant radiation therapy among breast cancer survivors. Breast. 2019;43:48-54.

53. James M, Swadi S, Yi M, Johansson L, Robinson B, Dixit A. Ischaemic heart disease following conventional and hypofractionated radiation treatment in a contemporary breast cancer series. J Med Imaging Radiat Oncol. 2018;62:425-31.

54. Killander F, Wieslander E, Karlsson P, Holmberg E, Lundstedt D, Holmberg $\mathrm{L}$, et al. No increased cardiac mortality or morbidity of radiation therapy in breast cancer patients after breast-conserving surgery: 20-year follow-up of the randomized SweBCGRT trial. Int J Radiat Oncol Biol Phys. 2020;107:701-9.
55. Kim DY, Youn JC, Park MS, Lee S, Choi SW, Ryu KH, et al. Cardiovascular outcome of breast cancer patients with concomitant radiotherapy and chemotherapy: A 10-year multicenter cohort study. J Cardiol. 2019;74:175-81.

56. Wennstig AK, Wadsten C, Garmo H, Fredriksson I, Blomqvist C, Holmberg L, et al. Long-term risk of ischemic heart disease after adjuvant radiotherapy in breast cancer: results from a large population-based cohort. Breast Cancer Res. 2020;22:10.

57. Wollschläger D, Merzenich H, Schwentner L, Janni W, Wiegel T, Bartkowiak D, et al. Self-reported long-term cardiac morbidity in breast cancer patients: a retrospective cohort study in Germany (PASSOS Heart Study). Breast Cancer Res Treat. 2017;163:595604.

58. Boekel NB, Duane FK, Jacobse JN, Hauptmann M, Schaapveld M, Sonke GS, et al. Heart failure after treatment for breast cancer. Eur J Heart Fail. 2020;22:366-74.

59. Chou YH, Huang JY, Kornelius E, Chiou JY, Huang CN. Major adverse cardiovascular events after treatment in early-stage breast cancer patients receiving hormone therapy. Sci Rep. 2020;10:1408.

60. Lee J, Hur H, Lee JW, Youn HJ, Han K, Kim NW, et al. Longterm risk of congestive heart failure in younger breast cancer survivors: A nationwide study by the SMARTSHIP group. Cancer. 2020;126:181-8.

61. Lawrenson R, Lao C, Ali A, Campbell I. Impact of radiotherapy on cardiovascular health of women with breast cancer. J Med Imaging Radiat Oncol. 2019;63:250-6.

62. Leung HW, Chan AL, Muo CH. Late cardiac morbidity of adjuvant radiotherapy for early breast cancer - a population-based study. $\mathrm{J}$ Cardiol. 2016;67:567-71.

63. Killander F, Anderson H, Kjellén E, Malmström P. Increased cardio and cerebrovascular mortality in breast cancer patients treated with postmastectomy radiotherapy -25 year follow-up of a randomised trial from the South Sweden Breast Cancer Group. Eur J Cancer. 2014;50:2201-10.

64. Clarke M, Collins R, Darby S, Davies C, Elphinstone P, Evans V, et al. Effects of radiotherapy and of differences in the extent of surgery for early breast cancer on local recurrence and 15year survival: an overview of the randomised trials. Lancet. 2005;366:2087-106.

65. Ragaz J, Olivotto IA, Spinelli JJ, Phillips N, Jackson SM, Wilson KS, et al. Locoregional radiation therapy in patients with high-risk breast cancer receiving adjuvant chemotherapy: 20-year results of the British Columbia randomized trial. J Natl Cancer Inst. 2005;97:116-26.

66. Woodward WA, Strom EA, McNeese MD, Perkins GH, Outlaw EL, Hortobagyi GN, et al. Cardiovascular death and second non-breast cancer malignancy after postmastectomy radiation and doxorubicinbased chemotherapy. Int J Radiat Oncol Biol Phys. 2003;57:327-35.

67. Højris I, Overgaard M, Christensen JJ, Overgaard J. Morbidity and mortality of ischaemic heart disease in high-risk breast-cancer patients after adjuvant postmastectomy systemic treatment with or without radiotherapy: analysis of DBCG $82 \mathrm{~b}$ and $82 \mathrm{c}$ randomised trials. Radiotherapy Committee of the Danish Breast Cancer Cooperative Group. Lancet. 1999;354:1425-30.

68. Gyenes G, Rutqvist LE, Liedberg A, Fornander T. Long-term cardiac morbidity and mortality in a randomized trial of pre- and postoperative radiation therapy versus surgery alone in primary breast cancer. Radiother Oncol. 1998;48:185-90.

69. Rutqvist LE, Liedberg A, Hammar N, Dalberg K. Myocardial infarction among women with early-stage breast cancer treated with conservative surgery and breast irradiation. Int J Radiat Oncol Biol Phys. 1998;40:359-63.

70. Rutqvist LE, Lax I, Fornander T, Johansson H. Cardiovascular 
mortality in a randomized trial of adjuvant radiation therapy versus surgery alone in primary breast cancer. Int $\mathrm{J}$ Radiat Oncol Biol Phys. 1992;22:887-96.

71. Jones JM, Ribeiro GG. Mortality patterns over 34 years of breast cancer patients in a clinical trial of post-operative radiotherapy. Clin Radiol. 1989;40:204-8.

72. Høst H, Brennhovd IO, Loeb M. Postoperative radiotherapy in breast cancer - long-term results from the Oslo study. Int J Radiat Oncol Biol Phys. 1986;12:727-32.

73. Hamood R, Hamood H, Merhasin I, Keinan-Boker L. Risk of cardiovascular disease after radiotherapy in survivors of breast cancer: A case-cohort study. J Cardiol. 2019;73:280-91.

74. Jacobse JN, Duane FK, Boekel NB, Schaapveld M, Hauptmann M, Hooning MJ, et al. Radiation dose-response for risk of myocardial infarction in breast cancer survivors. Int J Radiat Oncol Biol Phys. 2019;103:595-604.

75. Lee CH,Zhang JF, Yuan KS, Wu AT, Wu SY. Risk of cardiotoxicity induced by adjuvant anthracycline-based chemotherapy and radiotherapy in young and old Asian women with breast cancer. Strahlenther Onkol. 2019;195:629-39.

76. Wu SP, Tam M, Vega RM, Perez CA, Gerber NK. Effect of breast irradiation on cardiac disease in women enrolled in BCIRG-001 at 10-year follow-up. Int J Radiat Oncol Biol Phys. 2017;99:541-8.

77. Tan CH, Chao TT, Liu JC, Lin CH, Huang YS, Chang CM, et al. Breast cancer therapy and age difference in cardiovascular disease risks: a population-based cohort study in Taiwan. Taiwan J Obstet Gynecol. 2016;55:98-103.

78. Onwudiwe NC, Kwok Y, Onukwugha E, Sorkin JD, Zuckerman IH, Shaya FT, et al. Cardiovascular event-free survival after adjuvant radiation therapy in breast cancer patients stratified by cardiovascular risk. Cancer Med. 2014;3:1342-52.

79. Hooning MJ, Botma A, Aleman BM, Baaijens MH, Bartelink H, Klijn JG, et al.Long-term risk of cardiovascular disease in 10-year survivors of breast cancer. J Natl Cancer Inst. 2007;99:365-75.

80. Geiger AM, Chen W, Bernstein L. Myocardial infarction risk and tamoxifen therapy for breast cancer. Br J Cancer. 2005;92:1614-20.

81. Drost L, Yee C, Lam H, Zhang L, Wronski M, McCann C, et al. A systematic review of heart dose in breast radiotherapy. Clin Breast Cancer. 2018;18:e819-24.

82. Hong TS, Ritter MA, Tomé WA, Harari PM. Intensity-modulated radiation therapy: Emerging cancer treatment technology. Br J Cancer. 2005;92:1819-24.
83. Yeboa DN, Evans SB. Contemporary breast radiotherapy and cardiac toxicity. Semin Radiat Oncol. 2016;26:71-8.

84. Bergom C, Currey A, Desai N, Tai A, Strauss JB. Deep inspiration breath hold: Techniques and advantages for cardiac sparing during breast cancer irradiation. Front Oncol. 2018;8:87.

85. Simonetto C, Eidemüller M, Gaasch A, Pazos M, Schönecker S, Reitz D. Does deep inspiration breath-hold prolong life? Individual risk estimates of ischaemic heart disease after breast cancer radiotherapy. Radiother Oncol. 2019;131:202-7.

86. Duma MN, Baumann R, Budach W, Dunst J, Feyer P, Fietkau R, et al. Heart-sparing radiotherapy techniques in breast cancer patients: a recommendation of the breast cancer expert panel of the German Society of Radiation Oncology (DEGRO). Strahlenther Onkol. 2019;195:861-71.

87. Mulliez T, Veldeman L, Speleers B, Mahjoubi K, Remouchamps V, Van Greveling A, et al. Heart dose reduction by prone deep inspiration breath hold in left-sided breast irradiation. Radiother Oncol. 2015;114:79-84.

88. Formenti SC, Gidea-Addeo D, Goldberg JD, Roses DF, Guth A, Rosenstein BS, et al. Phase I-II trial of prone accelerated intensity modulated radiation therapy to the breast to optimally spare normal tissue. J Clin Oncol. 2007;25:2236-42.

89. Yue NJ, Goyal S, Park JH, Jones S, Xu X, Khan A, et al. Optimization of heart block in the left-sided whole breast radiation treatments. Front Oncol. 2014;4:342.

90. Welsh B, Chao M, Foroudi F. Reducing cardiac doses: a novel multi-leaf collimator modification technique to reduce left anterior descending coronary artery dose in patients with left-sided breast cancer. J Med Radiat Sci. 2017;64:114-9.

91. Brana I, Zamora E, Oristrell G, Tabernero J. Cardiotoxicity. In: Dicato MA, Van Cutsem E, editors. Side Effects of Medical Cancer Therapy: Prevention and Treatment. 2nd ed. London: Springer International Publishing; 2018: p 367-406.

92. Jones LW, Haykowsky MJ, Swartz JJ, Douglas PS, Mackey JR. Early breast cancer therapy and cardiovascular injury. J Am Coll Cardiol. 2007;50:1435-41.

93. Jones RL, Ewer MS. Cardiac and cardiovascular toxicity of nonanthracycline anticancer drugs. Expert Rev Anticancer Ther. 2006;6:1249-69.

94. Chu TF, Rupnick MA, Kerkela R, Dallabrida SM, Zurakowski D, Nguyen L, et al. Cardiotoxicity associated with tyrosine kinase inhibitor sunitinib. Lancet. 2007;370:2011-9. 